Originalveröffentlichung in: B. Seidensticker-- M. Vöhler.(Hg.), Gewalt und Ästhetik. Zur. Gewalt und ihrer. Darstellung in der griechischen Klassik, Berlin 2006, S. 223-257

Barbara E. Borg (Exeter)

\title{
Gefährliche Bilder? Gewalt und Leidenschaft in der archaischen und klassischen Kunst
}

Krieg und Gewalt sind Themen, die seit einigen Jahren auch in der Diskussion antiker Denkmäler, Verhaltensweisen und Wertvorstellungen eine zentrale Position einnehmen. ${ }^{1}$ In diesem Zusammenhang ist in letzter Zeit von archäologischer Seite öfter auf ein interessantes Phänomen hingewiesen worden: In der attischen Vasenmalerei zeichnet sich in den Jahren nach etwa 480 v. Chr. ein deutlicher Wandel in der Darstellung von Gewalt ab. $\mathrm{Da}$ dieser Wandel bereits vielfach beschrieben worden ist, ${ }^{2}$ kann ich

1 Frühere Versionen dieses Beitrags konnte ich bei verschiedenen Gelegenheiten in Bonn, Heidelberg, Tübingen und Berlin vorstellen und diskutieren. Mein besonderer Dank gilt dem Arbeitskreis, Bild und Gesellschaft im klassischen Griechenland‘, von dessen Anregungen ich sehr profitiert habe, sowie den Veranstaltern des Kolloquiums „Gewalt und Ästhetik: Gewalt und Formen der Gewaltdarstellung in der griechischen Klassik".

2 Ioanna Mennenga: Untersuchungen zur Komposition und Deutung hometischer Zweikampfszenen in der griechischen Vasenmalerei, Diss. Berlin 1976; Manuela Papadakis: Ilias- und Ilioupersisdarstellungen auf frühen rotfigurigen Vasen, Frankfurt a. M. u. a. 1994; Christian Ellinghaus: Aristokratische Leitbilder-Demokratische Leitbilder. Kampfdatstellungen auf attischen Vasen in archaischer und klassischer Zeit, Münster 1997; Brigitte Knittlmayer: Die Attische Aristokmatie und ihre Helden. Darstellungen des trojanischen Sagenkreises im 6. und frühen 5. Jahrhundert v. Chr., Heidelberg 1997; Florens Felten: Blutdurst oder Verhaltensmuster. Zur Bedeutung der Kampfbilder in der archaischen und klassischen griechischen Kunst. In: Steine und Wege. Festschrift für Dieter Knibbe, hrsg. von Peter Scherrer et al., Wien 1999, S. 195-9; Meret Mangold: Kassandra in Athen. Die Eroberung Trojas auf attischen Vasenbildern, Berlin 2000; Matthias Recke: Gewalt und Leid. Das Bild des Krieges bei den Athenern im 6. und 5. Jh. v. Chr., Istanbul 2002; Tonio Hölscher: Images of War in Greece and Rome: Between military practice, public memory and cultural symbolism. In: Journal of Roman Studies 93 (2003) S. 1-17; Günter Fischer und Susanne Moraw: Die andere Seite der Klassik. Gewalt im 5. und 4. Jahthundert v. Chr. Kulturwissenschaftliches Kolloquium Bonn, 11.-13. Juli 2002, Stuttgart 2005, passim mit reicher Bibliogtaphie in den einzelnen Beiträgen. 
mich hier auf einige Beispiele beschränken, die den Befund illustrieren sollen:

Sportliche Agone können in archaischer Zeit zumindest auf Symposionsgeschirr in ihrer ganzen realen Blutigkeit und Brutalität wiedergegeben werden, während man seit etwa 490 weniger drastische Darstellungen, vor allem den Beginn oder das Ende des Kampfs, bevorzugt. ${ }^{3}$ Archaische Bilder des ,privaten' Bereichs des Symposions zeigen Gewalt, etwa sexuelle Gewalt, meist gegen Frauen, oder Gewalttätigkeit der Zecher und Komasten untereinander, und auch diese Bilder werden in der frühen Klassik abgelöst durch eher beschauliche Darstellungen. ${ }^{4}$ Die größten Grausamkeiten in Darstellungen finden sich jedoch im Kontext mythischer Kämpfe und Kriege, insbesondere des Trojanischen Krieges, ohne daß diese Taten im Bild immer erkennbar negativ gewertet wären ${ }^{5}-$ auf diesen Punkt komme ich zurück.

Um 480/70 verschwinden die meisten dieser Szenen aus dem Repertoire. Bezeichnenderweise erscheint etwa die Tötung des Troilos nach ca. 490 nur noch in einer unsicheren Darstellung, die Schleifung Hektors bald nach 500 und der tödliche Zweikampf mit Achill nach 480 überhaupt nicht mehr. ${ }^{6}$ Andererseits stellt die Anwendung von Gewalt während des gesamten hier betrachteten Zeitraums, also auch in klassischer Zeit, durchaus ein nahe-

3 Martin Bentz: Spiel um Leben und Tod? Gewalt und Athletik in klassischer Zeit. In: Fischer und Moraw (2005) [Anm. 2], S. 129-11; Adrian Stähli: Die Rhetorik der Gewalt in Bildern des archaischen und klassischen Griechenland. In: Fischer und Moraw (2005) [Anm. 2], S. 28-32; beide mit weiterer Bibliographie.

$4 \mathrm{Zu}$ Gewalttätigkeiten der Zecher untereinander s. Ezio Pellizer: Outlines of a morphology of Sympotic entertainment. In: Sympotica. A Symposium on the Symposion, hrsg: von Oswyn Murray, Oxford 1984, S. 177-84, bes. S. 181-82 mit Abb. 16; Michael Vickers: Greek Symposia, London o. J. [1978], S. 16. 20 Abb. 21. 22; Stähli (2005) [Anm. 3], S. 24-28 mit Abb. 2-3. Zu Gewalt gegen Frauen Martin Kilmer: Sexual violence. Archaic Athens and the recent past. In: Owls to Athens. Essays on classical subjects presented to Sir Kenneth Dover, hrsg. von Elisabeth M. Craik, Oxford 1990, S. 261-77. Zum Symposion allgemein Alfred Schäfer: Unterhaltung beim griechischen Symposion. Darbietungen, Spiele und Wettkämpfe von homerischer bis in spätklassische Zeit, Mainz 1997, passim, der allerdings die groberen Umgangsformen auf die Ausweitung des Symposions auf breitere Bevölkerungsschichten zurückführen möchte (ebd. S. 67), eine Erklärung, die angesichts der Entwicklung der Bilder anderer Themenbeteiche kautn befriedigen kann.

5 Stähli (2005) [Anm. 3], S. 33-43; Ralf von den Hoff: ,Achill, das Vieh“? Zur Problematisierung transgressiver Gewalt in klassischen Vasenbildern. In: Fischer und Moraw (2005) [Anm. 2], S. 225-46. 
liegendes und natürliches Mittel zur Durchsetzung von Zielen dar, ${ }^{7}$ und Gewalttaten sind ein zentrales Motiv fast aller Tragödien. ${ }^{8}$

Ich möchte im folgenden versuchen, diesen Befund besser zu verstehen. Vorausschicken möchte ich, daß hier ein pragmatischer, selektiver und aus moderner Sicht gewählter Gewaltbegriff zugrunde gelegt wird, der eine kollektive Bezeichnung für alle physischen Verletzungen bis zur Tötung eines anderen Menschen darstellt. Hierin macht sich auch eine moderne Fragestellung bemerkbar, die an antiken Problematisierungsweisen und Diskursen teilweise vorbeizielt, aber gerade deshalb aufschlußreich sein kann. Auch braucht bei der Komplexität des Themas wohl kaum betont zu werden, daß meine Thesen einen sehr vorläufigen Charakter besit-

6 Zur Tötung des Troilos s. IIMC I, Zürich und München 1981, S. 80-90. 94-5 s. v. Achilleus (Anneliese Kossatz-Deissmann); IIMC VIII, Zürich und Düsseldorf 1997, S. 92-94 s.v. Troilos (Anneliese Kossatz-Deissmann); von den Hoff (2005) [Anm. 5]. Zur Schleifung Hektors s. IIMC I, Zürich und München 1981, S. 13940; 145-6 s.v. Achilleus Nr. 585-600 (Anneliese Kossatz-Deissmann); Recke (2002) [Anm. 2] S. 72-4; 291-2 mit Diagramm 9.1-2; Zum Zweikampf Achill Hektor LIMC ebd. S. 133-5; 137-8 Nr. 559-70; s. zu Zweikämpfen der Helden vor Troja auch Recke ebd. 11-20; 258-67 mit Diagramm 1.1-4. Einzig der Zweikampf zwischen Achill und Memnon läuft noch einige Zeit weiter, wird jedoch durch die Darstellung der Schicksalswägung in den Hintergrund gedrängt, die offenbar im eigentlichen Interesse stand. Zur Deutung der Wägeszene s. Christian Kunze: Dialog statt Gewalt. Neue Erzählperspektiven in der frühklassischen Vasenmalerei. In: Fischer und Moraw (2005) [Anm. 2], S. 66-71; vgl, auch Knittlmayer (1997) [Anm. 2], S. 100-8 mit leicht abweichender Deutung.

7 s. u. mit Anm. 39 und 40.

8 Entsprechend ist auch die Sekundärliteratur zum Thema unüberschaubar. Genannt seien hier nur wenige neuete Arbeiten, welche grundsätzlichere Überlegungen zur Gewalt in Tragödien anstellen: Anne P. Burnett: Revenge in Attic and Later Tragedy, Berkeley, CA u. a. 1998; William V. Harris: Restraining Rage. The Ideology of Anger Control in Classical Antiquity, Cambridge MA u. a. 2001, S. 161-74; Elizabeth S. Belfiore: Murder among friends. Violation of philia in Greek tragedy, Oxford 2000; Ruth Padel: Whom Gods destroy. Elements of Greek and tragic madness, Princeton, NJ 1995; Kleopatra Ferla: Von Homers Achill zur Hekabe des Euripides. Das Phänomen der Transgression in der griechischen Kultur, München 1996: Diese wichtige Arbeit ist mir leider zu spät bekannt geworden als daß ich sie im folgenden noch hätte berücksichtigen können. Andrea Ercolani: Gewalt in der griechischen Tragödie. In: Fischer und Moraw (2005) [Anm. 2], S. 89-101 ist unergiebig, da er ausschließlich nach moralischer und philosophischer Reflexion sucht und so zu dem Schluß kommt: „In den uns ethaltenen Tragödien fehlt eine ausführliche Behandlung der Gewaltproblematik vom Standpunkt der moralischen und philosophischen Reflexion." (Ebd. S. 95) 
zen. Mir geht es zunächst darum, zwei verschiedene Felder oder Aspekte näher zu beleuchten, die in Hinblick auf die Frage relevant sein dürften: 1) Funktionen und Motivationen physischer Gewaltanwendung und ihre Akzeptanz innerhalb der Gesellschaft, und 2) die spezifischen Möglichkeiten und Grenzen von Bildern (im Gegensatz zu verbalen Äußerungen), bestimmte Inhalte auszudrücken.

\section{Physische Gewalt als Problemlösungsstrategie}

Seit in der spätgeometrischen Zeit erstmals narrative und mythologische Bilder auf griechischen Vasen erscheinen, finden sich auch Darstellungen von blutigen Kämpfen. Trotz der schematischen Darstellungsweise der Zeit sind die Details der Verwundungen und Tötungsweisen in aller Deutlichkeit dargestellt (Abb. 1). ${ }^{9}$

Dies bleibt auch in der protokorinthischen und korinthischen Vasenmalerei so, welche die Brutalität des Krieges, die Verwundungen, Leichenfledderei usw. drastisch vor Augen führt (Abb. 2a-c). ${ }^{10}$

Ein ähnlicher Befund ergibt sich, wie bereits erwähnt, für die attischschwarzfigurige und frührotfigurige Vasenmalerei.

Dieser Befund verwundert keineswegs; nicht etwa, weil die betreffenden Epochen tatsächlich kriegerischer oder gewalttätiger gewesen seien als spätere, sondern weil die Darstellungen ganz in Übereinstimmung mit der Ideologie, mit den Normen und Wertvorstellungen der archaischen Gesell-

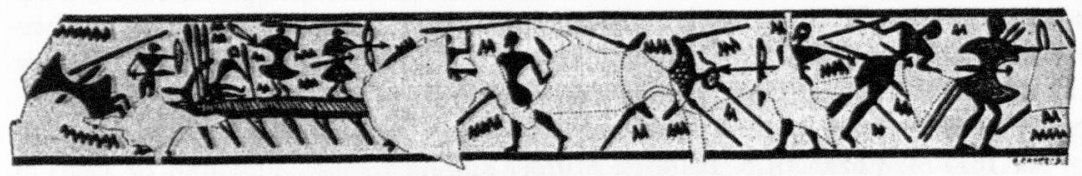

Abb. 1: Geometrische Oinochoe mit Kampfdarstellungen. Kopenhagen, Nationalmuseum.

9 John Boardman: Early Greek vase painting, $11^{\text {th }}-6^{\text {th }}$ centuries BC. A handbook, London 1998, Abb. 49-50. 65; Hilda L. Lorimer: The hoplite phalanx with special reference to the poems of Archilochus and Tyrtaeus. In: Annual of the British School in Athens 42 (1947), S. 77 Abb. 1.

10 Lorimer (1947) [Anm. 9], S. 98-102 Abb. 8c; 9a-d; auch Boardman (1998) [Anm. 9], Abb. 178,3; s. auch die Vorliebe für drastische Darstellungen auf Chalkidischen Gefäßen: Boardman (1998) [Anm. 9], Abb. 469. 471. 475. 482. 

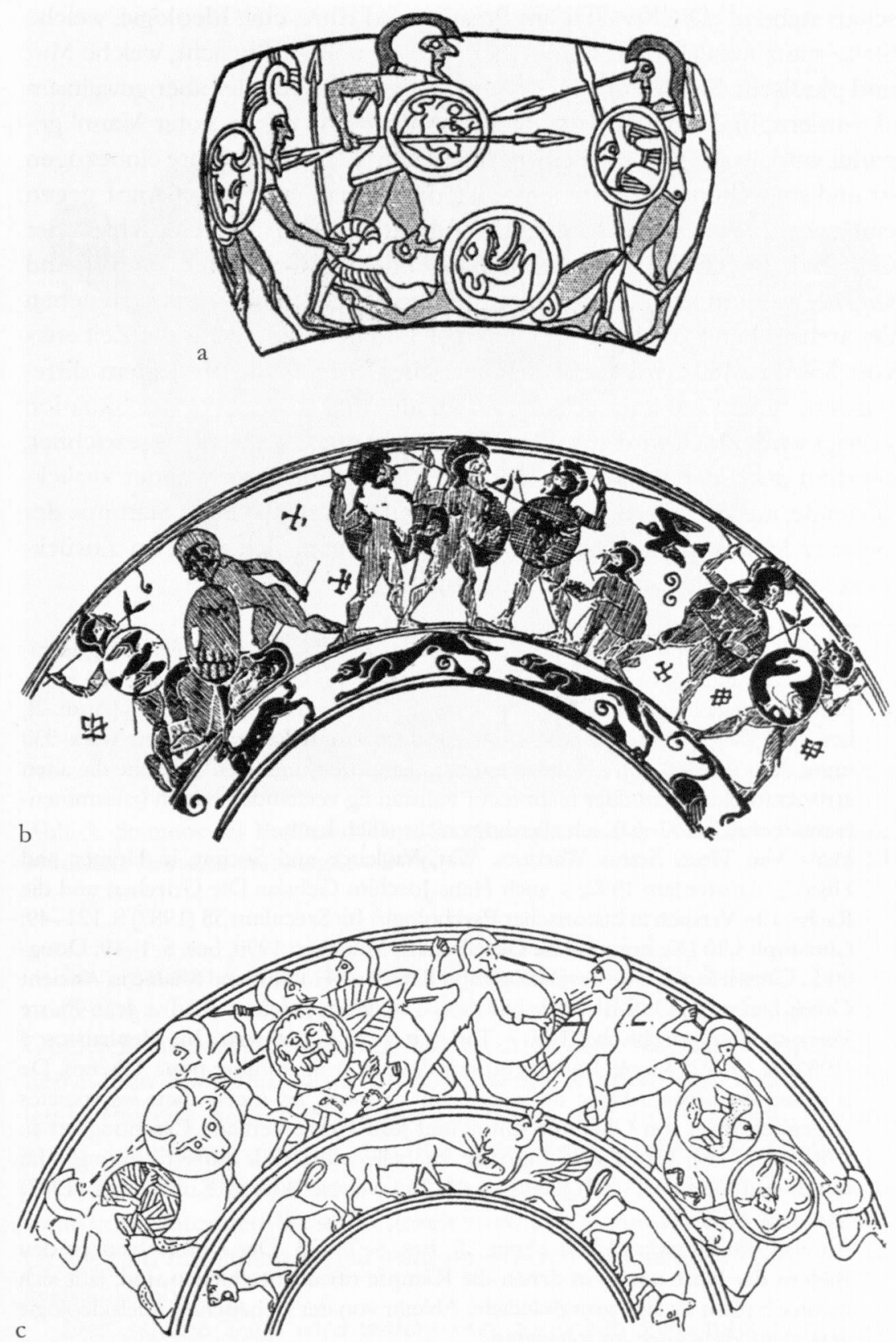

Abb. 2a-c: Protokorinthische Aryballoi mit Kampfdarstellungen. a) Paris, Louvre Inv. CA931; b) Paris, Louvre c) Syrakus, Museo Archeologico Regionale. 
schaft stehen. ${ }^{11}$ Die Rivalität um Prestige und Ehre, eine Ideologie, welche Status ganz wesentlich von der Anerkennung abhängig macht, welche Mut und physische Stärke entweder von sich aus auslösen oder aber gewaltsam einfordern, in der ein gewalttätiger Mann regelmäßig ein ,guter Mann' genannt wird, in der sogar der eigene Tod in den Handel um Ehre einbezogen ist und ein ,schöner Tod' derjenige ist, den man in tapferem Kampf gegen einen ebenbürtigen Gegner erleidet und durch den man im Gedächtnis der Gesellschaft weiterlebt, beherrschen nicht nur das Geschehen von Ilias und Odyssee, sondern auch die historische Wirklichkeit. ${ }^{12}$ Dies bestätigen neben der archaischen Lyrik auch die Denkmäler. In den Bildern aus der Zeit etwa von 530 bis 480 wird meist klar zwischen Sieger und Besiegtem differenziert, indem letzterer schwer verwundet und in auswegloser Situation gezeigt wird. Doch wird auf diese Weise nicht nur der Sieger ausgezeichnet, sondern auch der Besiegte. ${ }^{13}$ Der von Achill tödlich verwundet zurücksinkende, aus mehreren Wunden blutende Hektor auf einem Stamnos des Berliner Malers in München (Abb. 3) etwa scheint sich noch im Zurücksinken gegen seine Niederlage zu sträuben. ${ }^{14}$

11 Âhnlich Stähli (2005) [Anm. 3], S. 33-43 zu den Gewalttaten Achills auf griechischen Vasen. S. auch Michael Shanks: Art and the Greek City State. An interpretive archaeology, Cambridge 1999, bes. S. 107-13; Ellinghaus (1997) [Anm. 2], bes. S. 237-92, dessen These einer, "Tendenz zum Realismus“ seit etwa 530 unter dem Einfluß von „Neureichen bzw. Emporkömmlingen“, welche die alten aristokratischen Leitbilder nicht mehr vollständig verstanden hätten (zusammenfassend ebd. S. 257-61), ich allerdings nicht teilen kann.

12 Hans Van Wees: Status Warriors. War, Violence and Society in Homer and History, Amsterdam 1992; s. auch Hans-Joachim Gehrke: Die Griechen und die Rache. Ein Versuch in historischer Psychologie. In: Saeculum 38 (1987) S. 121-49; Christoph Ulf: Die homerische Gesellschaft, München 1990, bes. S. 1-49; Douglas L. Cairns: Aidos. The Psychology and Ethics of Honour and Shame in Ancient Greek literature, Oxford 1993, bes. S. 1-146. Zum ,schönen 'Tod' s. Jean-Pierre Vernant: Der griechische Tod - Tod mit zwei Gesichtern. In: Hephaistos 3 (1981) S. 17-22; Nicole Loraux: Mourir devant Troie, tomber pour Athènes. De la gloire du héros à l'idée de la cité. In: La mort, les morts dans les sociétés anciennes, hrsg. von Gherardo Gnoli und Jean-Pierre Vernant, Cambridge et al. 1982, S. 27-43; Jean-Pierre Vernant: La belle mort et le cadavre outragé. In: Gnoli und Vernant (1982) [ebd.], S. 45-76; weitere Bibl. in Knittlmayer (1997) [Anm. 2], S. 71-73.

13 Ähnlich Knittlmayer (1997) [Anm. 2], bes. S. 71-73. Der Unterschied zu den Bildern der Jahre davor, in denen die Kämpfe oft unentschieden sind, läßt sich demnach nicht als eine grundsätzliche Abkehr von der archaischen Adelsideologie verstehen; dazu auch im folgenden.

14 München, Antikensammlung 2406: $\mathrm{ARV}^{2}$ 207.137; $\mathrm{Add}^{2}$ 194; Para 343; CVA München (5) Taf. 238-9. 


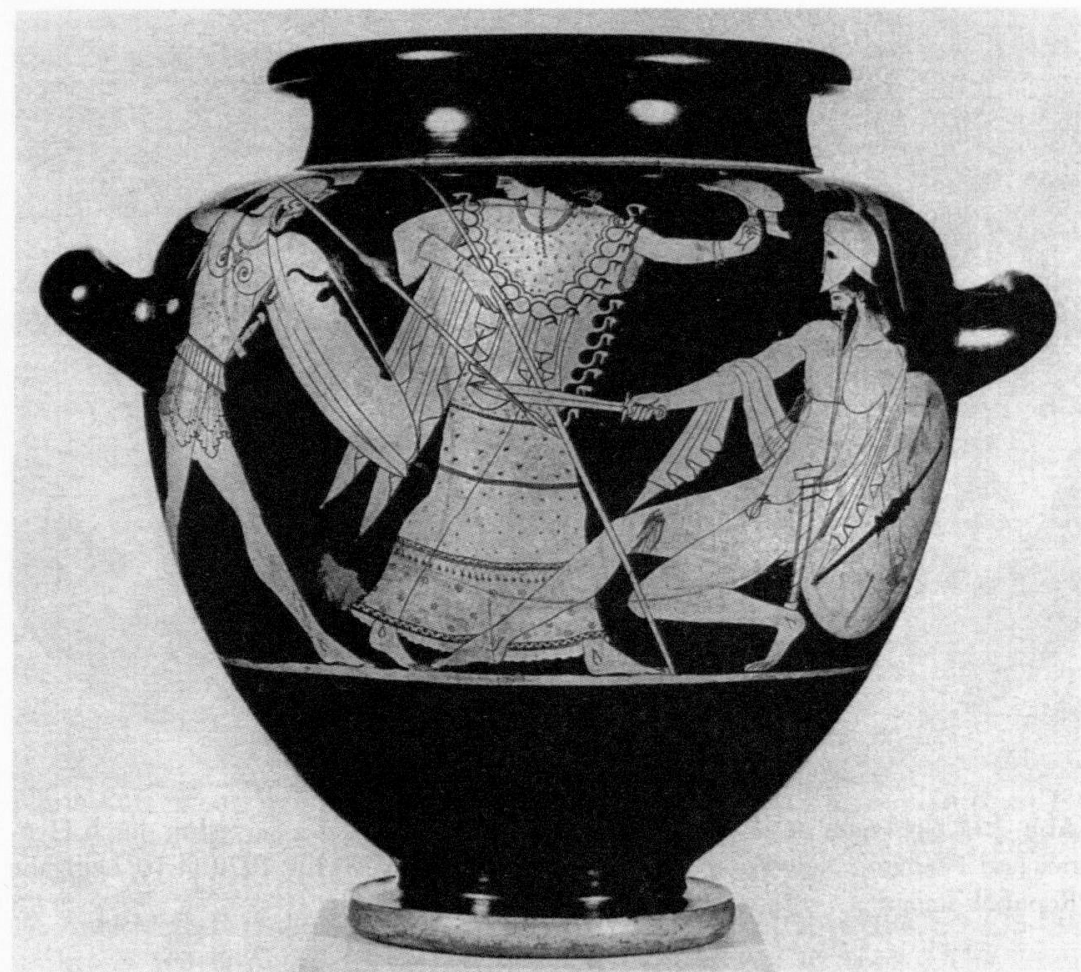

Abb. 3: Stamnos des Berliner Malers; Zweikampf zwischen Achilleus und Hektor. München, Antikensammlung Inv. 2406.

Sein ganzer Körper ist voller Spannung, und das Schwert hält er noch fest in der ausgestreckten Rechten gegen den Angreifer gerichtet. Obgleich zusammenbrechend, hat er doch den schönen Körper aller heroischen Kämpfer, ja, er ist durch seine Körpergröße und raumfüllende Präsenz dem Achill sogar überlegen, der im Vergleich beinahe etwas schmächtig und an den Rand gedrängt erscheint. ${ }^{15}$ Ähnlich ist auch der von Hypnos und Thanatos geborgene Leichnam Sarpedons zwar von blutenden Wunden gezeichnet, ansonsten aber in ,heroischer Größe und mit jedem Detail seines perfekten athletischen Körpers dargestellt (Abb. 4).

Der ,schöne Tote wird zudem von göttlichen Personifikationen geborgen, eine Ehrenerweisung, die seinen Ruhm und Status zusätzlich unterstreicht: Derjenige Held der Ilias, der vielleicht den Normen der home- 


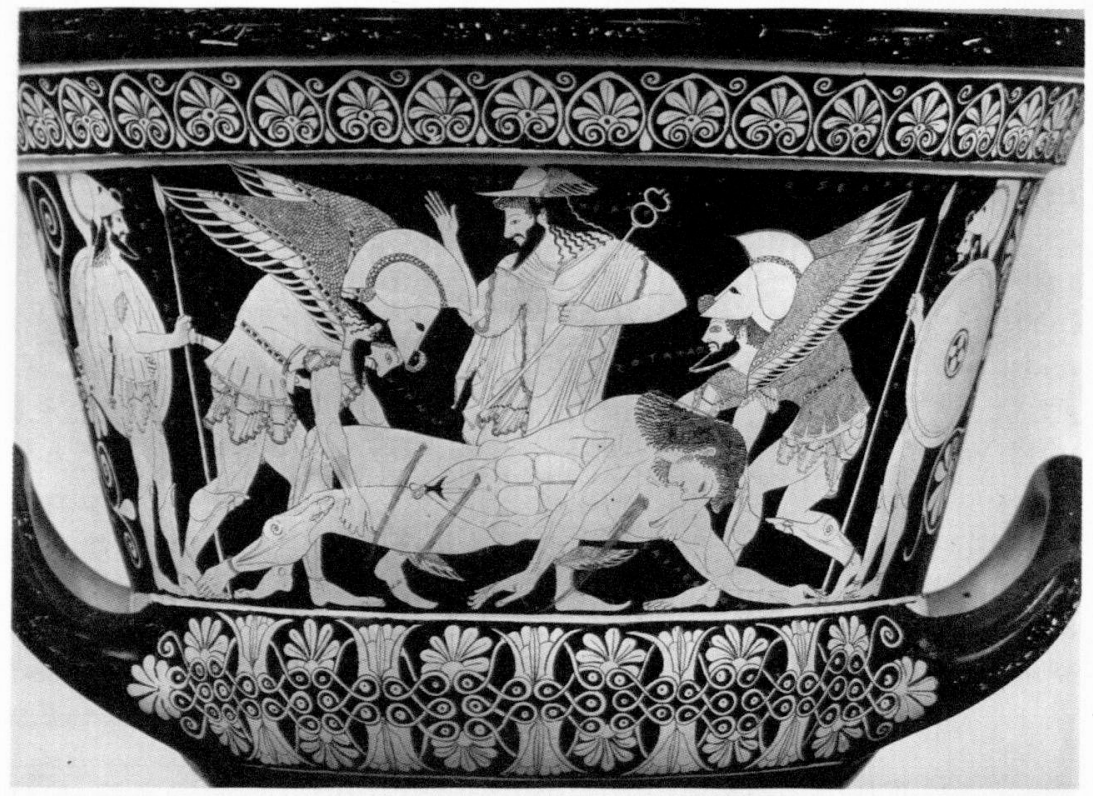

Abb. 4: Kelchkrater des Euphronios; Bergung der Leiche des Sarpedon durch Hypnos und Thanatos. New York, Metropolitan Museum of Art Inv. 1972.11.10; Leihgabe Republik Italien.

rischen Gesellschaft am besten entspricht, wird von Zeus durch einen schlafartigen Tod ohne Schrecken ausgezeichnet. ${ }^{16}$

15 Anders die Deutung von Susanne Muth: Zwischen Pathetisierung und Dämpfung. Kampfdarstellungen in der attischen Vasenmalerei des 5. Jhs. v. Chr. In: Fischer und Moraw (2005) [Anm. 2] S. 192-9, die offenbar davon ausgeht, es könne in den Kampfdarstellungen nur um den Sieger gehen, und die die Aufmerksamkeit, welche die Besiegten in den Darstellungen der fraglichen Zeit erhalten, als eine Art unerwünschten Nebeneffekt ansieht, den man mit den späteren Darstellungen auszugleichen gesucht hätte. Anders die klassischen Bilder, in denen der Unterlegene oft als zurückweichend oder gar fliehend dargestellt wird. Hier ist ihrer Deutung insoweit zuzustimmen, als nun tatsächlich der Sieger der alleinige Held (und die alleinige Identifikationsfigur) der Darstellung ist und die Überlegenheit zu einer absoluten wird.

16 H. Allan Shapiro: Personifications in Greek art. The representation of abstract concepts 600-400 B. C., Kilchberg und Zürich 1993, S. 132-48; Recke (2002) [Anm. 2], S. 60 4; 288 mit Diagramm 6.1-2; zur Symbolik Barbara E. Borg: Der Logos des Mythos. Allegorien und Personifikationen in der frühen griechischen Kunst, München 2002, S. 164-7. 
Eine ähnliche Vorstellung scheint auch den Kourosstatuen auf attischen Gräbern zugrunde zu liegen. ${ }^{17}$ Als besonders instruktives Beispiel mag hier das Grabmonument eines gewissen Kroisos aus Anavyssos dienen, der der Inschrift zufolge in einer unbekannten Schlacht in det vordersten Reihe starb (Abb. 5). ${ }^{18}$

Sein Bildnis zeigt ihn in seiner ganzen Schönheit und Jugend: die langen Locken, das lächelnde Gesicht als Ausdruck seiner Charis, der muskulöse Körper mit den mächtigen Schenkeln, sein fester Stand „,mit beiden Füßen gestemmt auf das Erdreich", das Inbild des idealen Kriegets und schönen Toten wie Tyrtaios es so oft beschreibt. ${ }^{19}$ Das Epigramm weist eine Reihe formaler Figenheiten auf, die ein gebildeter Zeitgenosse als Reminiszenz an epische und elegische Formulierungen erkennen konnte.

In der archaischen Gesellschaft spielten demnach physische Stärke und Kampfesmut als probate, ja, geradezu erwartete Mittel der Durchsetzung von Ansprüchen, zur Vergeltung von Beleidigungen aller Art und als Ausweis von Status und Größe zumindest der Ideologie nach eine wesentliche Rolle und waten den Dichtungen etwa des Alkaios, des Solon, des Theognis oder Xenophanes zufolge auch ein Faktum der realen Lebenswirklichkeit. Hans van Wees hat die archaischen Gesellschaften Griechenlands m.E. überzeugend mit der Mafia und ihrer Ideologie der Vendetta verglichen, ${ }^{20}$

17 Anna Maria D'Onofrio: Korai e kouroi funetari attici. In: AION 4 (1982), S. 135-70, bes. S. 163-8; Andrew F. Stewart: When is a kouros not an Apollo? The Tenea ,Apollo ${ }^{c}$ revisited. In: Corinthiaca. Studies in honor of Darrell A. Amyx, hrsg. von Mario A. Del Chiaro, Columbia 1986, S. 54-70, bes. S. 60-5; Christine SourvinouInwood: ,Reading 'Greek Death to the End of the Classical Period, Oxford 1995, S. 140-297, bes. S. 170-80. 221-78, die aber m. E. den Aspekt des Preises der Verstorbenen zugunsten persönlicher Trauer über den Verlust unterschätzt.

$18 \mathrm{Zu}$ ärchaischen Grabepigrammen s. Joseph W. Day: Rituals in Stone. Early Greek grave epigrams and monuments. In: Journal of Hellenic Studies 109 (1989) S. $17-40$, S. 20 zur Kroisos-Basis.

19 „Drum auf! im Spreizschritt halt’ man stand, mit beiden Füßen gestemmt ans

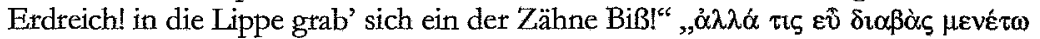

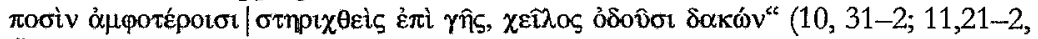
Übers. Joachim Latacz); s. auch 10.21-32; 11; 12 West; vgl. Hom. Il. 22.71-73: „Einem jungen Mann, der gefallen, steht alles an, auch wenn er vom scharfen Erze zerrissen daliegt; und alles ist schön an dem Toten, was da erscheinet." (Übers. Roland Hampe); s. auch Hölscher (2003) [Anm. 2], S. 11: ,The fighting wartiors in battle scenes ate kouroi set into motion".

20 Hans van Wees: The mafia of early Greece. Violent exploitation in the seventh and sixth centuries BC. In: Organised Crime in Antiquity, hrsg. von Keith Hopwood, London 1999, S. 1-51; Hans van Wees: Megara's mafiosi. Timocracy and violence in Theognis. In: Alternatives to Athens, hrsg. von Roger Brock und S. Hodkinson, Oxford 2000, S. 52-67. 
a
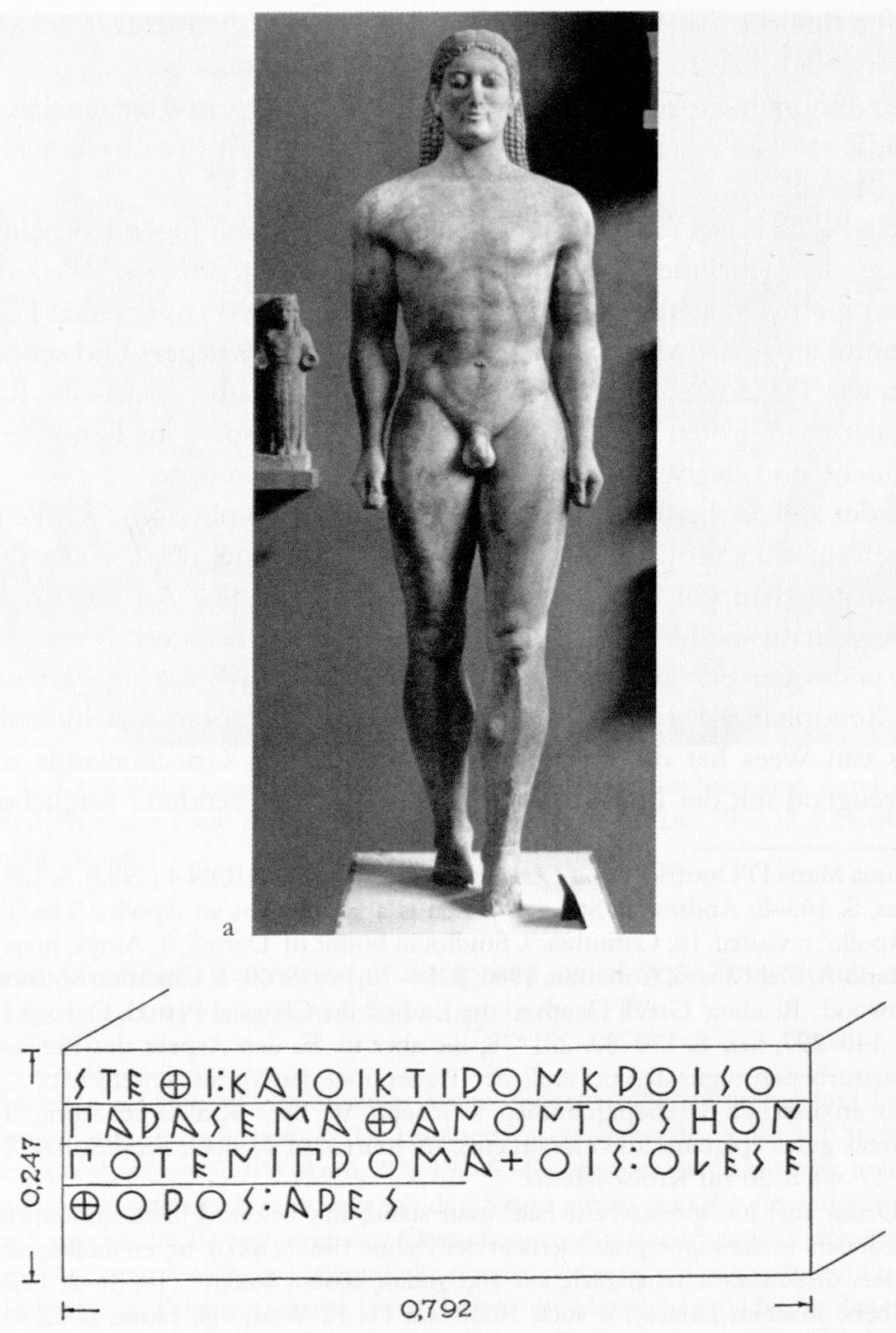

Fig. 1

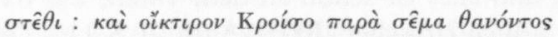

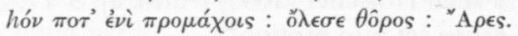

" Stay and mourn at the monument of dead Kroisos,

b whom furious Ares destroyed in the front ranks."

(Richter)

Abb. 5a,b: a) Grabstatue des Kroisos; b) Zeichnung, Transkription und Übersetzung der Basisinschrift (G. Richter). Athen, Nationalmuseum Inv. 3851. 
auch wenn man über das tatsächliche Ausmaß an Gewalttaten im Zusammenhang des archaischen Elitenwettbewerbs streiten kann.

Diese Ideologie wird jedoch bereits in archaischer Zeit problematisch, wie etwa schon Hesiods Unterscheidung zwischen der guten und schlechten Eris zeigt (Erga 11-26). ${ }^{21}$ In der archaischen Lyrik beginnt ein expliziter Hybrisdiskurs, in dem auch Gewaltanwendung eine Rolle spielt. ${ }^{22}$ Doch steht hier nicht so sehr die Gewalt selbst im Zentrum, sondern das zentrale Problem jeder auf Ehre und Anerkennung gegründeten Gesellschaftsform: Hybris als eine Haltung, die geprägt ist durch eine fehlgeleitete und überzogene Vorstellung von sich selbst und der eigenen Stellung in der Welt, durch ein unangemessenes Gefühl der Überlegenheit, durch die überzogene Sorge um die eigene Ehre, die entscheidender wird als der Schaden und die Unehre, die man anderen antut. ${ }^{23}$ Insbesondere scheint sich in dieser Zeit der Topos des hybriden und in seiner Hybris auch zu überzogener oder unnötiger Gewalt neigenden Tyrannen herauszubilden, ein Topos, der im 5. Jh. sowohl bei Herodot und Thukydides als auch in der Tragödie voll entfaltet wird. ${ }^{24}$ Die außerordentliche Fixierung auf die Ehre des Finzelnen und seiner Familie bzw. seiner pbiloi, welche die Notwendigkeit, diese Ehre mit Gewalt zu verteidigen einschließt, führt zu Vendetta-artigen Fehden und einem Teufelskreis von Gewalt, welche die Gesellschaft zu sprengen drohen.

Als zentrales - wir würden sagen: psychologisches - Problem wird dabei der Zorn erachtet, der einerseits notwendige Voraussetzung für Kampfkraft und die Ausübung von Rache, ja, geradezu Ausweis der Arete und des Status des Helden ist, andererseits aber die Gefahr in sich birgt, den Sinn für das rechte Maß an Rache zu verlieren und dadurch Gegenrache zu provozieten. ${ }^{25}$ "If anger is wrong, then, this is not because it is morally wrong,

21 Michael Gagarin: The Ambiguity of eris in the Works and Days. In: Cabinet of the Muses. Essays on Classical and Comparative Literature in Honor of Thomas G. Rosenmeyer, hrsg. von Mark Griffith und Donald J. Mastronarde, Atlanta 1990, S. $173-83$.

22 Nicolas R. E. Fisher: Hybris. A study in the values of honour and shame in ancient Greece, Warminster 1992, bes. S. 201-46 zur archaischen Lyrik.

23 Fisher (1992) [Anm. 22], bes. S. 7-150; dazu mit wichtigen Ergänzungen Douglas L. Cairns: Hybris, Dishonour, and Thinking Big. In: Journal of Hellenic Studies 116 (1996) S. 1-32.

24 Fisher (1992) [Anm. 22], S. 247-452; s. auch Harris (2001) [Anm. 8], S. 157-200.

25 Harris (2001) [Anm. 8], passim, bes. S. 131-200; Leonard C. Muellner: The Anger of Achilles. Menis in Greek Epic, Ithaca, N.Y. u. a. 1996; Burnett (1998) [Anm. 8]; Douglas L. Cairns: Anger and Honour in Homer's Iliad. In: Ancient Anger; Petspectives from Homer to Galen, hrsg. von Susan M. Braund and Glenn W. Most, Cambridge et al. 2003, S. 11-49; Glenn W. Most: Anger and pity in Homer's Iliad. In: Ebd. S. 50-75. 
but because it may have unpredictable and unwelcome side-effects. " ${ }^{26} \mathrm{Die}$ drakonischen Gesetze, die Reformen des Solon usw, waren Versuche, diese Auswirkungen der Racheideologie einzudämmen, ohne allerdings die Ideologie grundsätzlicher in Frage zu stellen, und William Harris vermutet gar, daß die Prominenz der Ilias, in welcher der Zorn ein zentrales Thema darstellt, mit aktuellen Diskussionen während der Polisbildung um 700 und der Notwendigkeit zur Schaffung von Regeln des Zusammenilebens zusammenhängt. ${ }^{27}$

Auch in Bildwerken finden sich gelegentlich Hinweise auf die problematischen Aspekte des Krieges und der Gewalt. Wie ich an anderer Stelle ausführlicher zu zeigen gesucht habe, stellt die von Pausanias beschriebene sogenannte Kypseloslade, ein eigentlich zylindrisches, in fünf Registern mit Bildern dekoriertes Behältnis, welches die Kypseliden ins Heiligtum nach Olympia gestiftet hatten, ein solches - in seiner Art allerdings bislang einzigartiges - Beispiel dar. ${ }^{28}$ Die überwiegende Mehrzahl der Szenen handelt von verschiedenen Formen gewalttätiger Auseinandersetzungen, von denen die meisten sich problemlos in das bekannte Spektrum einfügen. Einige bemühen sich jedoch um einen ausdrücklich kritischen Blick. Ich greife hier nur den Zweikampf zwischen Agamemnon und Koon über der Leiche des Iphidamas heraus, der im 11. Buch der Ilias, der Aristic des Agamemnon, beschrieben wird $(11,248-63)$, wo er u. a. dazu dient, den Anführer der Achäer zu charakterisieren. ${ }^{29}$ Die Tötung von Iphidamas und Koon ist hier nur vordergründig als Heldentat geschildert, genau genommen aber ein fatales Ereignis. Weder Iphidamas und Koon selbst noch ein Familienmitglied oder Freund haben Agamemnon je geschadet: im Gegenteil, ihr Vater Antenor beherbergte einst die Gesandtschaft des Menelaos und Odysseus bei sich (Il. 3,203 ff.), rettete die beiden vor Übergriffen der Trojaner und sprach sich als Gegner der Parispartei in der Ratsversamm-

26 Van Wees (1992) [Anm. 12], S. 135. So auch noch der Tenor von Athenas Rede in Aischylos' Eumeniden 858-66.

27 Harris (2001) [Anm. 8], S. 27. 131-56.

28 Paus. 5,17,5-19,10; Barbara E. Borg: Epigrams in archaic art. The ,Chest of Kyp-

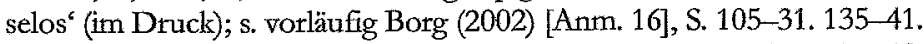

29 Vgl. Agathe Thornton: Homer's Iliad. Its Composition and the Motif of Supplication. Hypomnemata 81, Göttingen 1984, S. 78-80; Keith Stanley: The Shield of Homer. Narrative Structure in the Ikad, Princeton, NY 1993, S. 129; Samuel E. Bassett: The "Apoptio of Achilles. In: Transactions and Proceedings of the American Philological Association 65 (1934), S. 47-69; Oliver Taplin: Agamemnon's Role in the Iliad. In: Charakterization and Individuality in Greek Literature, hrsg: von Christopher Pelling, Oxford 1990, S. 60-82. 
lung für die Rückgabe der Helena aus (Il. 7,347ff.). ${ }^{30}$ Aus diesem Grund wird er auch bei der Eroberung Trojas gerettet. Der Ignoranz des Agamemnon entspringt seine unangemessene Grausamkeit, die im fraglichen Kampf noch dadurch gesteigert wird, daß er Koon, welcher den Leichnam seines Bruders bergen will, nicht nur tötet, sondern über diesem Leichnam auch noch köpft. ${ }^{31}$

Interessanterweise nimmt die Darstellung auf der Kypseloslade ausdrücklich dieselbe Perspektive ein, und zwar nicht nur durch die Wahl dieses ansonsten uninteressanten und in der griechischen Kunst singulären Zweikampfes. Eine Beischrift macht deutlich, daß es hier nicht in erster Linie um Agamemnons, sondern um Koons Kampf geht („Iphidamas ist

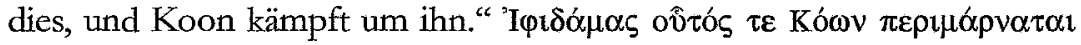

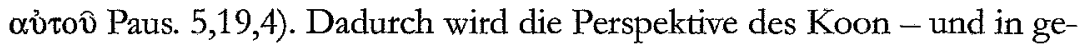
wisser Weise die des Iphidamas - zur Betrachterperspektive erhoben. Ein zweiter Hexameter bezieht sich auf die Darstellung auf dem Schild des Agamemnon und verweist den Betrachter auf die Symbolik des Schildzeichens: „Dies ist Phobos für die Sterblichen, der ihn trägt, Agamemnon."

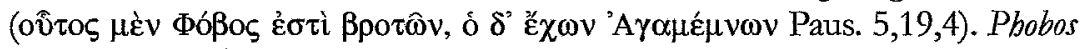
ist nicht nur ein Schildzeichen, er ist ein Attribut des Agamemnon. Das Epigramm enthält keinerlei Hinweis darauf, daß es sich um eine Darstellung handelt, es bezieht Phobos vielmehr direkt auf diesen sich durch besondere Grausamkeit auszeichnenden Helden. Pbobos ist sowohl die Fähigkeit des $\varphi$ oßeiv (,Scheuchens) als auch die Flucht bzw. der die Flucht auslösende psychische Zustand der Furcht und des Schreckens. ${ }^{32}$ Das Attribut Pbobos, das Agamemnon gewissermaßen einsetzen kann wie seinen Schild, kennzeichnet diesen somit als einen, der wie Ares phobos, die Fähigkeit des

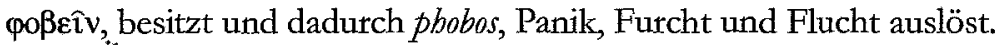

Die beiden Hexameter weisen demnach auf zwei wesentliche Aspekte der Episode hin, die deren Bewertung durch den Betrachter im Sinne der Ilias lenkt und, so würde ich behaupten, zu einem Kommentar des Krieges

30 Vgl. Thornton (1984) [Anm. 29], S. 79-80. Zur Analyse der Zweikämpfe s. auch mit etwas anderem Fazit Gisela Straßburger: Die kleinen Kämpfer der Ilias, Diss. Frankfurt a. M. 1954, S. 69-72.

31 Chatles Segal: The Theme of the Mutilation of the Corpse in the Iliad, Leiden 1971, S. 20-21; Vernant (1982) [Anm. 12]; Jon E. Lendon: Homeric vengeance and the outbreak of Greek wars. In: War and violence in ancient Greece, hrsg. von Hans van Wees, London 2000, S. 1-30, bes. S. 6-11.

32 Joachim Gruber; Über einige abstrakte Begriffe des frühen Griechischen, Meisenheim am Glan 1963, S. 15-39. Hartmut Exbse: Untersuchungen zur Funktion der Götter im homerischen Epos, Berlin/New York 1986, S. 29-34, betont einseitig den passiven Aspekt der Flucht und Furcht. 
insgesamt erweitert. Indem der eine Hexameter die Namen - und somit die Persönlichkeiten - der Opfer und nicht etwa des Siegers betont, wird an „die ganze Bitternis des Krieges ${ }^{\iota 33}$ erinnert, und Phobos ist der panische Furcht auslösende Schrecken und Terror des Kampfes und Krieges selbst, dem Koon und Iphidamas erliegen bzw. erlegen sind und den sie durch ihren Tod bestätigen. ${ }^{34}$

In einem anderen Bild wird - wiederum eine singuläre Darstellung - der Brudermord von Eteokles und Polyneikes dargestellt, vielleicht das Paradigma der Gefahren, welche im Wettbewerb um Ehre und Macht lauern. ${ }^{35}$ Wie ausführlicher gezeigt werden müßte, stellt die Kypseloslade inhaltlich wie in ihren Ausdrucksmitteln in mancher Hinsicht ein Äquivalent, möglicherweise sogar eine gezielte Reaktion auf den ps.-hesiodeischen Schild des Herakles dar, der, wie verschiedentlich argumentiert wurde, ebenfalls die problematischen Seiten archaischer Kriege thematisiert. ${ }^{36}$

Ein entscheidender ideologischer Wandel scheint in Athen aber erst mit der Vertreibung der Tyrannen und den ersten demokratischen Reformen einzusetzen. Zwar ist schon zuvor die Idee verbreitet, daß Hybris besonders als Ergebnis eines Zuviel an Macht oder Reichtum stasis hervorruft, wenn sie nicht vom Gesetz in Schach gehalten wird, ${ }^{37}$ doch scheint diese Erkenntnis erst mit Einrichtung der Demokratie allmählich zu konsequenten Veränderungen zu führen. Das Individuum tritt nun stärker in die Gemeinschaft zurück, der Ideologie einer Verteidigung der Ehre und Demon-

33 Straßburger (1954) [Anm. 30], S. 127.

34 Vgl. auch Thornton (1984) [Anm. 29], S. 79: ,The pathos of the blind destructiveness of war, suggested by the, glcaming chest ${ }^{\prime}$ of the first pair killed, by the second pair having been caught and ransomed before, by the third pair dooming themselves to death by their very appeal for mercy, reaches its climax in Agamemnon's killing the sons of Antenor in ignorance. “- Es versteht sich aber wohl von selbst, daß damit keine grundsätzliche Kritik am Krieg geübt oder eine pazifistische Haltung ausgedrückt werden sollte.

35 Borg (2002) [Anm. 16], S. 109-11.

36 William G. Thalmann: Conventions of Form and Thought in Early Greek Epic Poetry, Baltimore 1984, S. 62-4. Als Appell für ein friedliches, geregeltes Zusammenleben, das die Schilddarstellungen dominiert, liest F. Graf mit Verweis auf Vergils Interpretation auch den Schild des Achill in der Ilias: Fritz Graf: Ekphrasis: Die Entstehung der Gattung in der Antike. In: Beschreibungskunst - Kunstbeschreibung. Ekphrasis von der Antike bis zur Gegenwart, hrsg. von Gottfried Boehm und Helmut Pfotenhauer, München 1995, S. 150-51. Zur Kypseloslade s. vorläufig Borg (2002) [Anm. 16], S. 105-31. 135-41.

37 Fisher (1992) [Anm. 22]; Nicholas R. Fisher: Hybris, tevenge and stasis in the Greek city-states. In: War and violence in ancient Greece, hrsg, von Hans van Wees, London 2000, S. 83-123. 
stration von Macht durch individuelle physische Stärke und Gewaltanwendung tritt die Vorstellung einer durch die Gemeinschaft legitimierten Rache zur Seite. Durch das ganze 5. Jh. hindurch bleiben die negativen Auswirkungen übersteigerter Selbstbezogenheit und Gier nach time bis hin zur Hybris, die sich u. a. in überzogenen Gewaltakten, vorzugsweise gegenüber der eigenen Familie oder den philoi, und in Verletzung ritueller Bräuche äußern, allgemeines und sorgsam gepflegtes Kliché. ${ }^{38}$

Andererseits bleibt jedoch die göttliche wie die soziale Ordnung der Athener auf Zorn und Rache gegründet, die Herrschaft des Zeus ebenso wie die Athenische Demokratie, welche der Idee nach einem blutigen Racheakt zu verdanken ist und in der die Gesetze nicht etwa einen Ersatz für Rache darstellten, sondern den Versuch, die Rache in Bahnen zu lenken, welche möglichen Schaden für den Rächer (und die Polis) vermeiden. ${ }^{39}$ Insofern kann L.C. Muellner mit gewissem Recht schreiben, menis sei ,not just an emotional state. It is a sanction meant to guarantee and maintain the integrity of the world order. ${ }^{640}$ Aischylos’ Eumeniden geben ein ebenso unmißverständliches Zeugnis von dem Bedürfnis nach Eindämmung von Stasis und Beendigung der Spirale von Racheakten durch Einsetzung eines Gerichtshofs (ausdrücklich z. B. 976-83) wie von der Akzeptanz des Zorns und des Wunsches nach Rache bei Ehrverletzung. Indem Athena die Eumeniden zudem auffordert, nicht mehr den Zorn der athenischen Bürger aufeinander anzuheizen, sondern den Zorn auf auswärtige Feinde zu lenken (858-65), legitimiert sie zudem explizit deren gewalttätige außenpolitische Aktivitäten.

38 Dies insbesondere in der Konstruktion der Tyrannenfigur, s. Harris (2001) [Anm. 8], S:-174-82; Richard Seaford, Tragic Tyranny. In: Popular Tyranny: Sovereignty and its discontents in ancient Greece, hrsg. von Kathrin A. Morgan, Austin 2003, S. $95-115$.

39 Gehrke (1987) [Anm. 12]; Burnett (1998) [Anm. 8]; Nicolas R. Fisher: Violence, masculinity and the law in classical Athens. In: When men were men. Masculinity, power and identity in classical antiquity, hrsg. von Lin Foxhall und John Salmon, London/New York 1998, S. 68-97; Fisher (2000) [Anm. 37]; Lendon (2000) [Anm. 31]; Danielle Allen: Angry bees, wasps, and jurors: the symbolic politics of ópy in in Athens. In: Braund und Most (2003) [Anm. 25], S. 76-98; noch Atistoteles akzeptiert Zorn im rechten $M a B$, „to be angry in the manner, at the things, and for the length of time that reason dictates." (Christopher Gill: Ancient Passions. Theories and Cultural Styles. In: The Literary Portrayal of Passion through the Ages, hrsg. von Keith Cameron, Lewiston, ME 1996, S. 1-10, hier: S. 2 mit Verweis auf Arist. NE 4,5); David Konstan: Aristotle on anger and the emotions. The strategies of status. In: Braund und Most (2003) [Anm. 25], S. 99-120.

40 Muellner (1996) [Anm. 25], S. 26. 

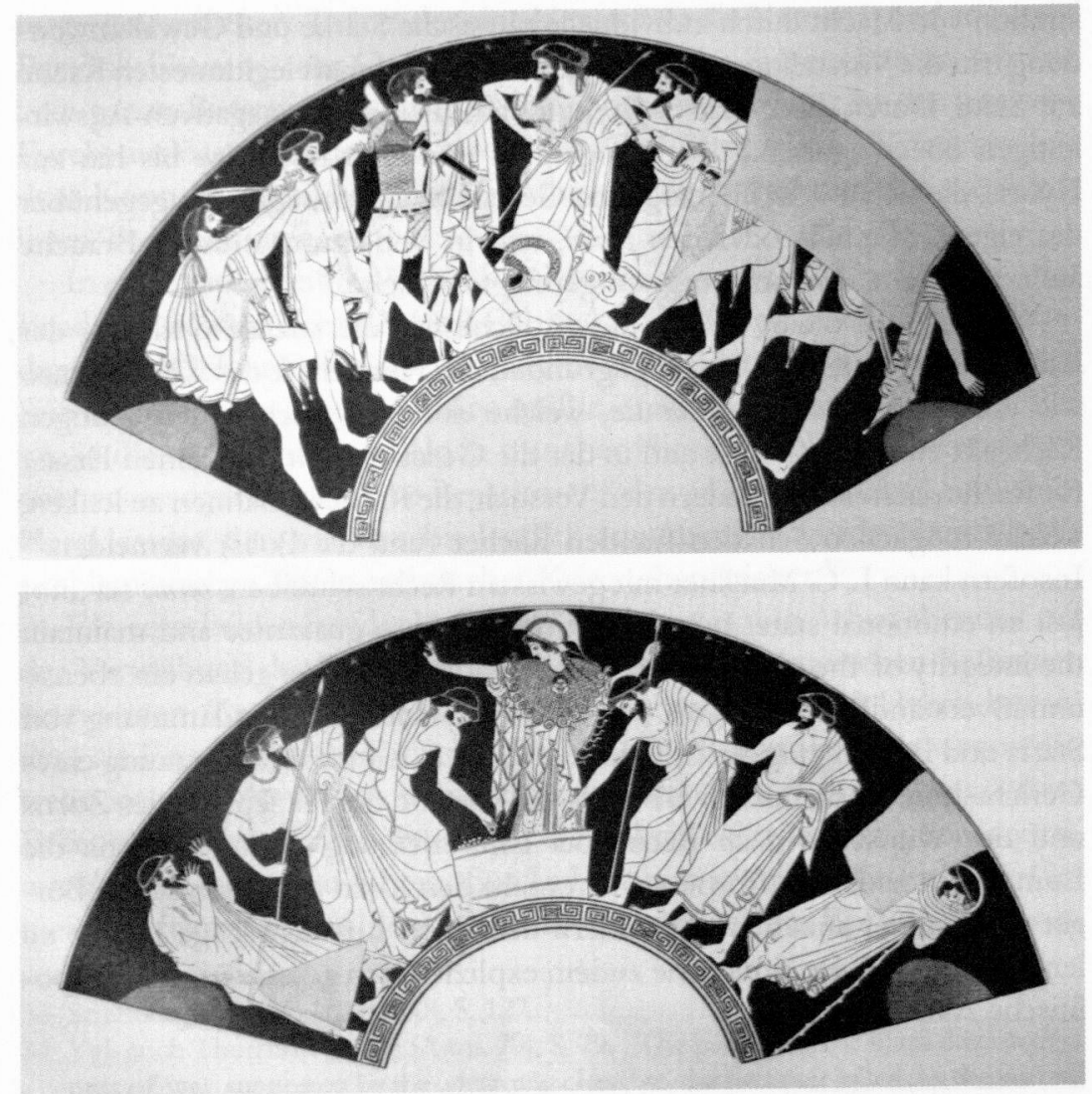

Abb. 6: Schale des Douris; Streit um die Waffen des Achill. Wien, Kunsthistorisches Museum Inv. 3695.

Diese kritische Auseinandersetzung mit den alten und neuen Ideologien scheint sich auch in den Bildern niederzuschlagen, und zwar bereits zu einer Zeit, die von den literarischen Quellen nur unzureichend abgedeckt wird. So sind die sich um 490 häufenden Darstellungen des Streits zwischen Ajas und Odysseus um die Waffen des Achill (Abb. 6) nicht so sehr als Zeugnis der Beliebtheit des Ajas im Zuge der Integration von Salamis in das Athenische Herrschaftsgebiet anzusehen, wie man gemeint hat, ${ }^{41}$ sondern als eine bildliche Parallele zu Aischylos' Eumenidendrama und damit als ein früher

41 Dyfri Williams: Ajax, Odysseus and the arms of Achilles. In: Antike Kunst 23 (1980), S. 143-44. 
Beleg für eine Diskussion der zentralen Thematik. Seit ca. 490 finden sich auf attischen Vasen zwei Versionen des Streits zwischen Odysseus und Ajas um die Waffen Achills. Die eine zeigt einen nur mühsam verhinderten blutigen Kampf zwischen den beiden, die andere eine Abstimmung. Und mehr noch, sechsmal werden beide Szenen, der bewaffnete Kampf und die Abstimmung, einander auf demselben Gefäß gegenübergestellt (Abb. 6) ${ }^{42}$

Die Darstellungen stimmen weder mit jener in der Aithiopis noch mit jener in der Kleinen Ilias überein, ${ }^{43}$ und man hat daraus auf eine verlorene Ajasdichtung als Vorlage geschlossen. ${ }^{44}$ Auch wenn dies, wie so oft, nicht völlig auszuschließen ist, scheint es mir ebenso gut möglich zu sein, daß die Vasenmaler die älteren Fassungen gezielt verändert haben, denn schon die Wahl des Themas, das als solches weit weniger Dramatik zu bieten hat als etwa der in der Folge dieser Entscheidung stehende Selbstmord des Ajas, ist bezeichnend und legt ein jenseits der reinen Erzählung liegendes Interesse an dem Thema nahe. Auf den Schalen jedenfalls sind es die Achäer selbst, die unter der Aufsicht Athenas abstimmen: traditionellen, ,heroischen' Problemlösungsstrategien wie dem blutigen Kampf werden zeitgemäßere Strategien wie Gerichtsverhandlung und Abstimmung gegenübergestellt. ${ }^{45} \mathrm{Daß}$ der in feinste Gewänder gehüllte und wohlfrisierte Agamemnon über den bewaffneten Konflikt wacht, während meist Athena die Abstimmung leitet, ist sicher kein Zufall. ${ }^{46}$ Gewaltsame, spontane Kon-

42 Zusammengestellt in LIMC I, Zürich und München 1981, S. $324-27$ s.v. Aias I (Odette Touchefeu); dazu Williams (1980) [Anm. 41], S. 137-45; Kunze (2005) [Antm, 6], S. 45-71, bes. S. 50-65.

43 Prokl. Exc. Aith. 29 f. (Davies); Aith. fr. 5 PEG; Prokl. Exc. IlMik. 2ff. (Davies); Schol. ap. Aristoph. Equ. 1056; IlMik. fr. 2-3 PEG.

44 Als bester Kandidat gilt hier Aischylos' Hoplon Krisis, doch s. hierzu Williams (1980) [Anm. 41], S. 143, und Kunze (2005) [Anm. 6], Anm. 40. Als Gegenargument ließe sich auch verwenden, daß in keiner erhaltenen Schriftquelle ein handgreiflicher Kampf zwischen den Helden erwähnt wird, das Bildschema des verhinderten Zweikampfes jedoch schon vor den hier in Rede stehenden Vasen bekannt ist: Kunze ebd., S. 61-63.

45 So auch die Deutung von Kunze (2005) [Anm. 6], S. 50-65; ähnlich schon Nigel Spivey: Psephological Heroes. In: Ritual, Finance, Politics. Athenian Demokratic Accounts Presented to David Lewis, hrsg. von Robin Osborne und Simon Hornblower, Oxford 1994, S. 39-51, aber s. auch hier Anm. 47. Bezeichnend auch die einzigartige Darstellung des Rededuells zwischen Odysseus und Ajas, das der Entscheidung wohl vorausgegangen sein muß: Kanze (2005) [Anm. 6], S. 51-53 mit Abb. 1.

46 Auf der Schale in Malibu, J. Paul Getty Museum 86.AE.286 (IIMC I, München und Zürich 1981, S. 325-26 s. v. Aias I Nr. 72; S. 83 [Odette Touchefeu]), scheint Agamemnon jedoch auch bei der Abstimmung den Vorsitz zu führen. 
fliktlösungen waren nicht nur ein Kennzeichen der homerischen Epen, sondern auch von Adelsgesellschaft, Timokratie und Tyrannis der archaischen Zeit, deren Oberschicht Agamemnon so sehr gleicht, während Athena eher für die Gemeinschaft der Polis steht, die hier zur Abstimmung schreitet. Insofern scheinen die Schalenbilder eine Diskussion zu spiegeln, welche so kurz nach der Abschaffung der Tyrannis und Einleitung demokratischer Reformen aktuelle politische Brisanz besaß.

Doch zweifellos handelt es sich hier nicht um ein Propagandawerk der Demokratie und nicht einmal um eine klare Stellungnahme zugunsten der neuen Verhältnisse. ${ }^{47}$ Noch bei Aischylos braucht es drei Tragödien und alles diplomatische Geschick Athenas, um die Entscheidung zugunsten eines Endes der Fehde herbeizuführen. Die Entscheidung, Achills Waffen dem Odysseus zu geben, galt in der Antike allgemein als ungerecht. ${ }^{48}$ Wo die Waffenstreitbilder mit einer weiteren Darstellung kombiniert werden, findet sich nur in einem Falle möglicherweise ein Hinweis auf einen positiven Effekt der Entscheidung, ${ }^{49}$ sonst aber auf den Tod des Ajas, dessen Bewertung letztlich dem Betrachter überlassen bleibt: Ist er die Folge übersteigerter Ehrsucht oder doch Ausweis der Ohnmacht auch demokratischer Entscheidungsfindungen, Gerechtigkeit zu erlangen? ${ }^{50}$

Auch andere Darstellungen der Zeit scheinen mir als Auseinandersetzung - und zwar als relativ ergebnisoffene Auseinandersetzung - mit den ideologischen und politischen Veränderungen der Zeit lesbar zu sein. Seit dem Ende des 6. Jhs. ist eine neue Vorliebe für mehrszenige Ilioupersisdarstellungen zu beobachten. ${ }^{51}$ Dies mag zunächst insofern bezeichnend sein, als die Eroberung Trojas hier trotz der Einzelepisoden mit

47 Anders Spivey (1994) [Anm. 45]; Kunze (2005) [Anm. 6], S. 63-65.

48 Pind. N. 7,22-7; 8,23-7; I. 4,53-7; dazu Roscher, ML I 1, Hildesheim 1884-86, Sp. 125-30 s. v. Aias Telamonios (C. Fleischer) mit weiteren Belegen.

49 So, falls im Innenbild der Schale des Douris in Wien tatsächlich die Übergabe der Waffen des Achill durch Odysseus an Neoptolemos dargestellt sein sollte; dazu Spivey (1994) [Anm. 45], S. 44 Anm. 4; Kunze (2005) [Anm. 6], Anm. 36.

50 Falls das Fragment einer Kylix des Douris (Vatikan 35092 Astatita Collection 133 mit MMA 1974.226) tatsächlich die Manipulation der Abstimmung zeigen sollte (Spivey 1994 [Anm. 45], S. 44. 50 Abb. 2,5), hätten wit hier einen weiteren Beleg für eine kritische Haltung gegenüber der neuen Form der Entscheidungsfindung. Von Manipulation handeln auch Pindar [s. hier Anm. 48] und Sophokles' Ajax. Euripides' Orestes (408 v. Chr.) endet in der Aporie: weder ein Gerichtsverfahren, noch Privatrache, noch die Götter können das Problem wirklich lösen: Fischer (1998) [Anm. 39], S. 83.

51 LIMC VIII, Zürich und Düsseldorf 1997, S. 650-57 s.v. Ilioupersis (Maria Pipili); Williams (1980) [Anm. 41]; von den Hoff (2005) [Anm. 5], S. 237-40. 


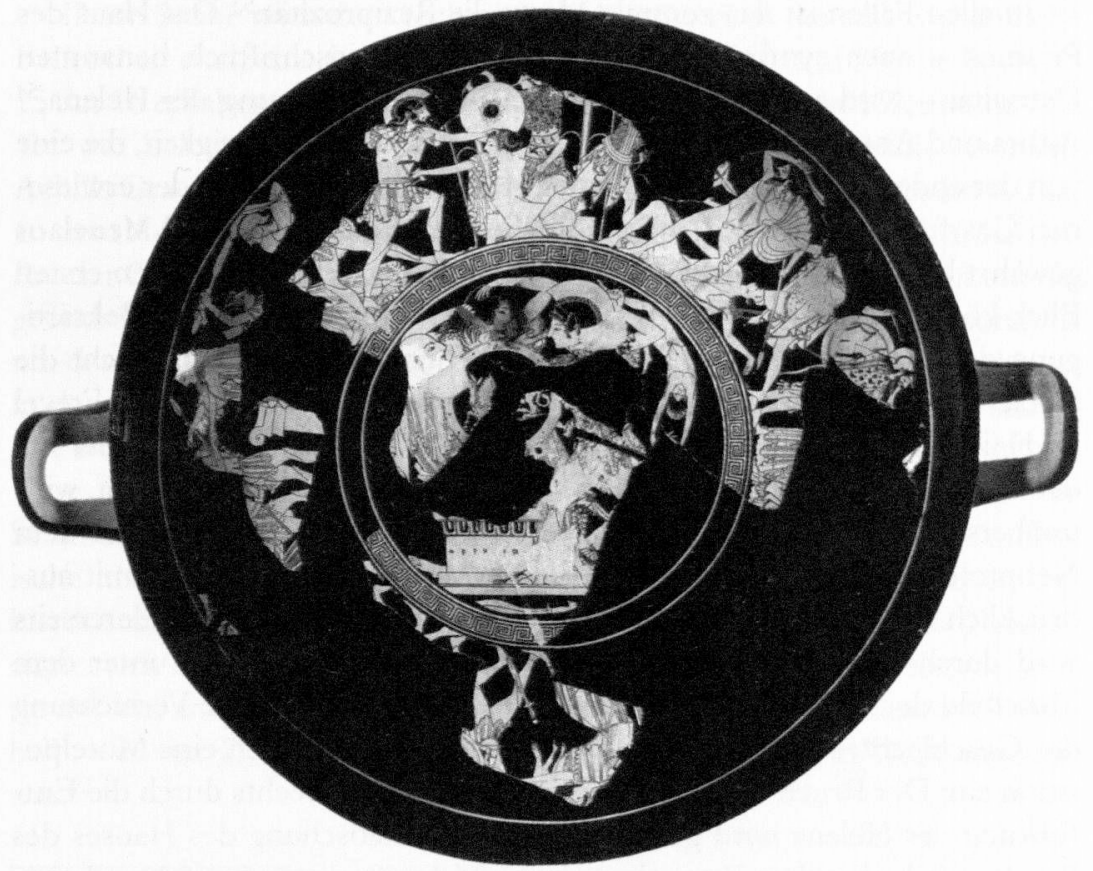

Abb. 7: Schale des Onesimos; Ilioupersis. Rom, Villa Giulia Inv. 121110.

ihren jeweiligen Protagonisten als Gemeinschaftsunternehmen erscheint, ein Eindruck, der durch die oftmals eingefügten anonymen Kampfgruppen noch zusätzlich unterstrichen wird. Wichtig ist jedoch auch die Wahl der neu hinzukommenden Szenen: Neben den Greueln der Eroberung und Rache finden sich nun auch Szenen der Rettung, manche zum ersten Mal in der Bildkunst. Onesimos beispielsweise hat in seiner Darstellung der Ilioupersis auf einer Schale in der Villa Giulia Ajas' Angriff auf Kassandra sowie die Ermordung von Astyanax und Priamos, vielleicht auch des Deiphobos, mit der Rückführung und Rettung der Helena, der Aithra sowie Theanos und Antenors kombiniert (Abb. 7). ${ }^{52}$

52 Rom, Villa Giulia 121110 (ehem. Malibu, J. Paul Getty Museum 83.AE.362): Dyfri Williams: Onesimos and the Getty Iliupersis. In: Greek Vases in the J. Paul Getty Museum 5 (1991), S. 41-64; Luca Giuliani: Bild und Mythos. Geschichte der Bilderzählung in der griechischen Kunst, München 2003, S. 211-14 Abb. 43; Michael J. Anderson: Onesimos and the interpretation of Ilioupersis iconography. In: Journal of Hellenic Studies 115 (1995) S. 130-35; ders.: The Fall of Troy in Early Greek Poetry and Art, Oxford 1997, S. $234-45$. 
In allen Fällen ist das zentrale Motiv die Reziprozität: ${ }^{53}$ Das Haus des Priamos - auch symbolisiert durch den eigens inschriftlich benannten Hausaltar - wird ausgelöscht als Rache für die Entführung der Helena, ${ }^{54}$ Aithra und Antenor profitieren von ausgleichender Gerechtigkeit, die eine von der endgültigen Rückfführung der Helena, der andere von der erwiesenen Gastfreundschaft und Schonung, die er einst Odysseus und Menelaos gewährt hat: gerettet werden nur die Freunde der Achäer. Auf den ersten Blick könnte man diese Kombination von Motiven demnach als Bekräftigung der Idee einer gerechten Rache ansehen. ${ }^{55}$ Doch dies ist nicht die ganze Wahrheit. An prominenter Stelle über dem Mittelbild ist Ajas' Frevel im Heiligtum der Athena dargestellt und durch die Inschrift HEPKEI $\Omega$ auf dem Altar, auf den sich Priamos vor seinem Angreifer geflüchtet hat, wird unübersehbar auf den heiligen Charakter des Ortes verwiesen, an dem Neoptolemos seine Rache vollzieht. Der Mord am Altar wird somit ausdrücklich der Tat des Ajas am Bild der Athena parallelisiert. Andererseits wird durch die Rückgewinnung Helenàs in derselben Achse unter dem Mittelbild der Anlaß des Krieges in Erinnerung gerufen. Die Vernichtung des Geschlechtes des Priamos nimmt somit auch inhaltlich eine Mittelposition ein: Der Bruch des von Zeus geschützten Gastrechts durch die Entführung der Helena wird gerächt durch die Auslöschung des Hauses des Frevlers, jedoch indem Zeus ein weiteres Mal mißachtet wird. ${ }^{56}$ Es scheint, als sei hier eines der Hauptdilemmata der griechischen Gesellschaft thematisiert worden. Auf der einen Seite die allgemeine Forderung nach und Akzeptanz von auch physisch vollzogener Rache, deren Maß die zuvor erlittene Beleidigung ist und welche als Notwendigkeit und Garant von Gerechtigkeit verstanden wird, ${ }^{57}$ auf der anderen die Gefahr, welche von dieser Rache ausgeht, indem sie dazu neigt, unkontrollierbar zu werden, und die notorische Schwierigkeit, das rechte $\mathrm{Maß} z u$ finden. ${ }^{58}$

53 Anderson (1995) [Anm. 52], bes. S. 134; Anderson (1997) [Anm. 52], bes. S. 244 45.

54 Die Kombination der Ermordung des Astyanax und des Priamos gegen die literarischen Varianten dient wohl dazu, die vollständige Auslöschung des Geschlechts des Priamos zu verdeutlichen; vgl. Williams (1991) [Anm. 52], S. 50-51.

55 So Williams (1991) [Anm. 52], S. 61, kritisch schon Anderson (1995) [Anm. 52], Anm. 47.

56 Teilweise ähnlich von den Hoff (2005) [Anm. 5], S. 237-40 zu anderen mehtszenigen Ilioupersisdarstellungen, deren Episoden er vielleicht zu Recht als eher kontrastierend aufeinander bezogen auffaßt.

57 Zum Motiv der Rache in der griechischen Außenpolitik s. Gehrke (1987) [Anm. 12]. 
Diese Suche nach dem rechten $\mathrm{Maß}$ könnte auch die außerordentliche Beliebtheit der Schleifung Hektors zwischen ca. 510 und 500 bedingen. ${ }^{59}$ 15 der 16 erhaltenen attischen Darstellungen fallen in diesen Zeitraum. Die Tat war bekanntlich schon in der Ilias umstritten; ${ }^{60}$ jedenfalls erscheint Achills Grausamkeit Apollon als Zeichen von Hybris, weshalb er am 9. Tag, an dem Achill den Leichnam um das Grab des Patroklos geschleift hat, im Rat der Götter für deren Eingreifen plädiert. Daß es auch hier nicht um die grundsätzliche Ablehnung der Schändung des Leichnams geht, sondern um das rechte $\mathrm{Maß}$, zeigt nicht nur das lange Zögern der Götter, überhaupt in das Geschehen einzugreifen, sondern vor allem die Diskussion selbst, in welcher das $\mathrm{Maß}$ an Schande, welche Hektor erleidet, gegen das Maß an Ehre aufgerechnet wird, welche Achill verdient. Diese Einstellung zur Schändung eines Leichnams ist grundsätzlich auch für die gesamte archaische und klassische Zeit gültig, wie verschiedentlich an literarischen wie historischen Quellen gezeigt worden ist. ${ }^{61}$ Zur Diskussion des Themas eignet sich die Episode der Ilias aber nicht nur deshalb besonders gut, weil sie auf ein anerkanntes literarisches Werk zurückgeht, sondern auch deshalb, weil der Zwiespalt der Bewertung bereits in diesem Werk angelegt war. $^{62}$

58 Dies ist letztlich auch der Konflikt, mit dem die Ilias beginnt und von dem Achills menis ihren Ausgang nimmt, und es ist zweifellos kein Zufall, daß ebendiese Szene auf der einen Außenseite der Onesimosschale dargestellt ist (Williams 1991 [Anm. 52], S. 56-58). In diesem Zusammenhang ist auch Gloria Ferraris Hinweis in Erinnerung zu rufen, daß die Griechen sowohl ihren Sieg über die Perser mit dem Sieg der Achäer über Troja gleichsetzen konnten, als auch die Frevel bei der Einnahme Trojas mit dem Frevel der Perser bei der Einnahme Athens, besonders der Akropolis. Offenbar war demnach die moralische bzw. ethische Bewertung der Ereignisse keineswegs eindeutig und, je nach Interessenlage, eine Identifizierung mit beiden Parteien möglich. Gloria Ferrari: The Ilioupersis in Athens. In: Harvard Studies in Classical Philology 100 (2000) S. $119-50$.

59 LIMC I (1981) [Anm. 6], S. 139-40. 145-6 Nr. 585-600; Recke (2002) [Anm. 2], S. 72-74. 291-92 mit Diagramm 9.1-2; von den Hoff (2005) [Anm. 5], S. 22728.

60 Hom. Il. 24,23-119; Segal (1971) [Anm. 31], S. 54-64.

61 Harris (2001) [Anm. 8], S. 129-30; zu Rache als Ehrengabe (geras) für den zuvor getöteten Freund oder Verwandten, auch in der Form der Verstümmelung von Gegnern auf dem Schlachtfeld, s. Lendon (2000) [Anm. 31], S. 6-11.

62 Auch von den Hoff (2005) [Anm. 5], S. 227-28 sieht in den Bildern der Schleifung den Widerstreit zwischen transgressiver Ehrverletzung und gerechter Rache datgestellt. Die Bilder scheinen mir jedoch unterschiedliches Gewicht auf den einen oder anderen Aspekt zu legen: s. im folgenden und hier Anm. 65. 


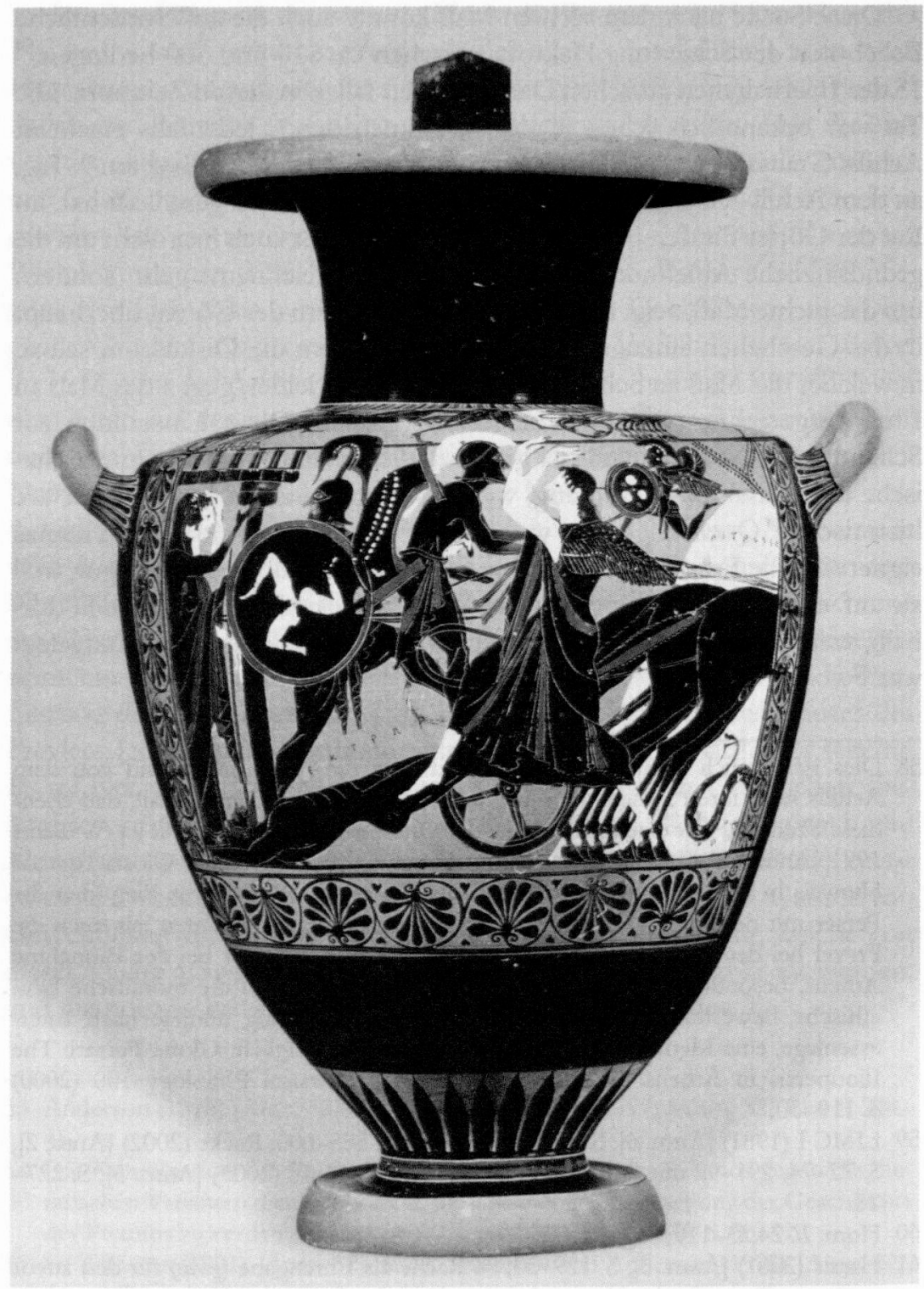

Abb. 8: Hydria der Leagros-Gruppe; Schleifung Hektors. Boston, Museum of Fine Arts Inv. 63.474. 
Ein Vasenmaler hat sich bemüht, diesen Zwiespalt noch deutlicher zum Ausdruck zu bringen. Eine Hydria der Leagros-Gruppe in Boston zeigt im Zentrum die Schleifung Hektors und rechts im Hintergrund den Grabhügel und das Eidolon des Patroklos (Abb. 8). ${ }^{63}$

Anders als auf den übrigen Gefäßen desselben Themas erscheinen hier zusätzlich am linken Bildrand die entsetzten Eltern Hektors. In der Regel hat man gemeint, es seien hier verschiedene Schleifungen, diejenige unmittelbar nach dem Sieg über Hektor von den Mauern der Stadt zum Lager der Griechen und diejenige um das Grab des Patroklos kombiniert worden. ${ }^{64}$ In gewisser Weise mag das zutreffen; eine solche ,Erklärung ${ }^{6}$ der Abweichung vom Text der Ilias verschleiert jedoch den Effekt einer solchen ,Kombination'. Entscheidend ist hier nicht die Identifizierung des Zeitpunktes, sondern der Versuch, das Geschehen in seiner ganzen ethischen Komplexität wiederzugeben: Während die Darstellung des Patroklosgrabes die Schleifung - als Ehrengabe für den Toten - mit der Idee einer grundsätzlich gerechtfertigten Rache für den Tod des Patroklos verknüpft, verweist schon die geflügelte Götterbotin im Zentrum des Bildes, welche wie in einigen anderen Bildern der Episode offenbar die Aufgabe der 'Thetis der Ilias erfüllt und dem Geschehen Finhhalt gebietet, auf das Urteil der Götter, das $\mathrm{Maß}$ gerechter Rache sei erfüllt. Ein Betrachter der Bostoner Hydria kann sich zudem veranlaßt fühlen, die Perspektive der Eltern zu berücksichtigen, welche die Greueltat des Achill wohl als unangemessen, in jedem Fall aber als furchtbares Schicksal und äußerste Erniedtigung etleben. ${ }^{65}$

Die Komplexität dieser spätarchaischen Bilder ist demnach beeindrukkend und die Zahl der Beispiele ließe sich leicht vermehren. ${ }^{66}$ Aber warum verschwinden diese Bilder nach ca. 480/70 aus dem Repertoire der Vasenmaler? Einige haben vermutet, die Erfahrung der Perserkriege hätte die

63 Boston, Museum of Fine Arts 63.474: LIMC I (1981) [Anm. 6], S. 139. 145 Nr. 586; Emily T. Vermeule: The vengeance of Achilles. The dragging of Hector at Troy. In: Bulletin of the Museum of Fine Arts 63 (1965) S. 138-59.

64 Ánneliese Kossatz-Deissmann in: LIMC I (1981) [Anm. 6], S. 139. 145 Nr. 586; ähnlich die Diskussionen in der übrigen Literatur, s. Bibl. ebd.

65 Hom. Il. 22,405ff. - Stemmer macht auf die Unterschiede zwischen den Darstellungen aufmerksam, die teils das Patroklosgrab und die Totenehrung, teils Achill und Hektor als Personen stärker in den Votdergrund stellen. Angesichts der zeitlichen Nähe aller Darstellungen zueinander scheint mir seine These einer chronologischen Entwicklung vom einen zum anderen Darstellungsinteresse schwer belegbar zu sein, auch wenn sie in das hier vorgetragene Erklärungsmuster passen würde. Seine Etklärung des Phänomens durch ein wachsendes ,Interesse am Szenischen" scheint mir jedoch zu kurz-zu greifen: Klaus P. Stähler: Grab und Psyche des Patroklos. Ein schwarzfiguriges Vasenbild, Münster 1967, S. 61. 
Wahrnehmung der Athener für die Schattenseiten von Krieg und Eroberung geschärft und zu mehr Mitgefühl für die Unterlegenen im Krieg und somit auch in den mythischen Kämpfen geführt. ${ }^{67}$ Richtig ist wohl, daß die mit der Eroberung Trojas einhergehenden Freveltaten der Achäer im 5. Jh. tatsächlich mit dem Frevel der Perser bei der Eroberung Athens und der Zerstörung des Heiligtums auf der Akropolis parallelisiert wurden. ${ }^{68}$ Doch kann diese Haltung kaum generalisiert werden, und es geht auch nicht um ,Mitgefühl', sondern um ethisch begründete Ansprüche. Dem widerspricht schon das Verhalten der Athener in ihren späteren Kriegszügen. Eher müßte man überlegen, ob nicht die Abkeht von trojanischen Themen, welche überwiegend die Perspektive der Achäer favorisiert hatten, gerade mit einer solchen Parallelisierung zwischen Ilioupersis und der Erstürmung der Akropolis zusammenhängen könnte. Eine Stütze würde diese Erklärung durch zwei trojanische Bildmotive erhalten, welche auch im 5. Jh. noch weiterlaufen, Ajas' Frevel an Athena und der Mord an Priamos am Altar des Zeus; denn auch in den entsprechenden Texten ist es besonders das Sakrileg der Schändung eines Heiligtums, welches die Eroberungen Athens bzw. Trojas miteinander verbindet. So erklärte sich möglicherweise, warum selbst die früher so beliebte Bergung des Leichnams des Achill nicht mehr dargestellt wird. Ohne Zweifel war die Bergung eines Leichnams in der Schlacht nach wie vor eine rühmliche Tat, aber wenn Achill zur problematischen Figur wird ${ }^{69}$ mag auch seine Leichenbergung nicht mehr uneingeschränkt

$66 \mathrm{DaB}$ auch ältere Bilder solche Komplexität gelegentlich erreichen, soll damit nicht in Abrede gestellt werden; s. z. B. Luca Giuliani: Kriegers Tischsitten, oder die Grenzen der Menschlichkeit. Achill als Problemfigur. In: Sinn (in) der Antike. Orientierungssysteme, Leitbilder und Wertkonzepte im Altertum, hrsg. von KarlJoachim Hölkeskamp et al., Mainz 2003, S. 135-61. Im übrigen scheint der Vorschlag von Recke (2002) [Anm. 2] grundsätzlich erwägenswert, besonders deutliche Darstellungen von Gewalt und deren Folgen im Sinne einer Problematisierung zu verstehen. Dabei kann es aber keinesfalls um eine „dezidierte Kritik am Krieg“ (S. 231; s. auch S. 94 und 235) unter dem Einfluß der Erfahrungen der Perserkriege gehen, die tait der Beruhigung der Bilder nach ca. 480/70 wieder überwunden sei, wie er meint. Dagegen spricht schon, daß die deutliche Zunahme an dargestellter Brutalität bereits deutlich vor den Persetkriegen beginnt. Zum grundsätzlichen Problem einer, Kritik am Krieg's. hier im folgenden mit Anm. 67.

67 Mangold (2000) [Anm. 2], bes. S. 148, zustimmend die Rezension von Susanne Moraw in: Gnomon 75 (2003), S. 340; Recke (2002) [Anm. 2], S. 231, S. 235; s. auch die Autoren in Muth (2005) [Anm. 15], Anm. 7; dazu Muth ebd. S. 188. 190 mit berechtigten methodologischen Einwänden.

68 Ferrati (2000) [Anm. 58].

69 Wie sich dies in den Bildern niederschlägt, zeigt von den Hoff (2005) [Anm. 5], S. 225-46; ebd. auch weitere Literatur zur Beurteilung Achills, wie sie sich aus schriftlichen Zeugnissen ergibt. 
positiv sein. Um die Jahrhundertmitte läßt ein Vasenmaler Achill erstmals sogar sterben. ${ }^{70}$ Vor diesem Hintergrund ist die andauernde Beliebtheit der Bergung des Sarpedon bezeichnend (Abb. 4), eines Motivs, das auf weißgrundigen Lekythen sogar auf normale Sterbliche übertragen wird. ${ }^{71}$

Darüber hinaus dürfte die Erfahrung der Perserkriege der durch die Abschaffung der Tyrannis und Eintichtung der Demokratie begonnenen Abkehr von der archaischen Ideologie einen entscheidenden Impuls gegeben haben. Die Abwehr der Perser war nur als Gemeinschaftsunternehmen möglich und erfolgreich, und es war vielleicht in diesen Kriegen, daß erstmals die Aufhebung der Rangunterschiede im gemeinsamen Kampf ihre ganze Wirkung entfaltete. ${ }^{72}$ So mag man vermuten, daß die alte Ordnung der Adelsgesellschaft nach den Perserkriegen für den größten Teil der Bevölkerung keine Attraktivität mehr besessen hat und damit auch die alten Bilder bis zu einem gewissen Grade obsolet wurden. ${ }^{73}$

70 Bochum, Ruhr-Universität, Antikentmuseum S 1060: LIMC I, Zürich und München 1981, S. 183 s. v. Achilleus Nr. 851 (Anneliese Kossatz-Deissmann); evtl. Achills Tod schon angedeutet auf einem Kyathos des frühen 5. Jhs. in London, British Museum E 808: LIMC ebd. Nr. 852.

71 Hier mit Anm. 16.

72 Lorimer (1947) [Anm. 9], S. 76-138; bes. Hans v. Wees: The development of the hoplite phalanx. Iconography and reality in the seventh century. In: War and violence in ancient Greece, htsg. von Hans van Wees, London 2000, S. 125-66; Johann P. Franz: Krieger, Bauern, Bürger. Untersuchungen zu den Hopliten der archaischen und klassischen Zeit, Frankfurt a. M. et al. 2002.

73 So etwa Stähli (2005) [Antn. 3], S. 33-43. Dem könnte man u. U. entgegen halten, $\mathrm{da}$ die Bilder nicht notwendigerweise die wahre Kampfweise der Zeit wiedergeben müssen, sondern der persönlichen Erfahrung des Kampfes, der letzllich doch immer in einem Zweikampf endete, Ausdruck verliehen (Knittmayer 1997 [Änm. 2], S. 68; Hölscher 2003 [Anm. 2], bes. S. 4-7, für die archaische Zeit, für die sich allerdings die zugrunde liegende These „combat in duels does not correspond to the reality of archaic warfare" [Hölscher ebd. S. 3] so kaum aufrecht erhalten läßt [vgl. hier Anm. 72]). Andererseits setzt sich in den sportlich-agonalen Bildern im 5. Jh. ebenfalls eine weitgehend gewaltfreie Ikonographie durch, ein Wandel, den Adrian Stähli mit der Ablehnung einer auf individuellen Prestigegewinn durch Gewalt setzenden archaisch-aristolstatischen Ideologie begründet (Stähli 2005 [Anm. 3], S. 28-32). Van Wees' interessante Überlegungen zum allmählichen Wandel des Selbstverständnisses des athenischen Adels von einer Kriegerklasse, in der die Bürger Waffen als Statussymbol und Zeichen ihrer Männlichkeit tragen, zu einer keisure-chass, die ihren Status durch Luxus demonstriert, tragen zum hier untersuchten Phänomen wohl nichts bei, jedenfalls dann nicht, wenn der Übergang tatsächlich schon um 530 einsetzt, wie Van Wees meint: Hans Van Wees: Greeks Bearing Arms. The State, the Leisure Class, and the Display of Weapons in Archaic Greece. In: Archaic Greece: New Approaches and New Evidence, hrsg; von Nicolas R. E. Fisher und Hans Van Wees, London 1998, S. 333-78. 
Aber selbst wenn man beide während der bzw. durch die Perserkriege sich vollziehenden Veränderungen - eine Abkehr von der alten Elitenideologie und eine neue Sicht auf das Schicksal der Trojaner - als wichtige Faktoren für den Wandel der Bildthemen akzeptiert, erklärt dies nicht, warum man die Darstellung von Episoden aus dem Trojanischen Krieg und weiterer Gewalttaten gleich ganz aufgegeben hat. Schließlich lebt das Thema in der Tragödie - und nicht nur dort - durchaus weiter. Dies könnte die Vermutung nahelegen, auch die spezifischen Bedingungen des Mediums der Vasenmalerei hätten zum Verschwinden von bis dahin so beliebten Themen beigetragen.

\section{Die Grenzen der Darstellungsmöglichkeiten}

Bilder haben, so scheint mir, tendenziell einen affirmativen Charakter. Sie konstatieren nicht nur ein Ereignis, sondern suggerieren durch Identifikationsangebote ein Einverständnis mit dem Dargestellten. ${ }^{74}$ Sofern sie einen kohärenten Situationsrahmen vorstellen, lassen sie das Geschehen plausibel und sinnvoll erscheinen. ${ }^{75}$ Nur wo das Dargestellte deutliche Signale der Kritik enthält oder ganz offensichtlich allen Normen und Regeln widerspricht, wird diese Affirmation durchbrochen oder schlägt gar ins Negative um, in die Distanzierung von den Protagonisten des Geschehens. Wenn beispielsweise auf der Schale des Brygos-Malers im Louvre (Abb. 9) ${ }^{76}$ aus-

$74 \mathrm{Zu}$ antiken Außerungen, nach denen Bilder ihre Betrachter zut Nachahmung anregen s. Renaud Robert: Ars regenda amore. Séduction érotique et plaisir esthétique: De Praxitèle à Ovide. In: Mélange de l'Ecole Française de Rome, Antiquité 104 (1992), S. 373-437, bes. S. 410-20; Adrian Stähil: Die Verweigerung der Lüste. Erotische Gruppen in der antiken Plastik, Berlin 1999, S. 36-37 Anm. 18. 20-22. Stähli (2005) [Anm. 3], S. 19-20 mit Anm. 2, möchte diese antiken Belege jedoch nicht auf die Wirkung von Bildern im allgemeinen, sondern auf ihr erotisches Genre beziehen. Dies scheint mir jedoch wenig plausibel, nicht zuletzt vor dem Hintergrund seiner eigenen Bildanalyse; dazu in folgenden.

75 Stähli (2005) [Anm. 3], S. 19-44, „Die Bilder produzieren Gewalt nicht, aber sie ordnen sie Sinn stiftend in einen Handlungskontext ein, der Gewalt als selbstverständliche und angemessene soziale Interaktionsform hinstellt und ihrer Ausführung ein plausibles Handlungsdrehbuch unterlegt." (ebd. S. 23)

76 Paris, Louvre G 152: ARV² 369,1; LIMC I, Zürich und München 1981, S, 772 s.v. Andromache I Nr. 46 (Odette Touchefeu-Meynier); LIMC II, Zürich und München 1984, S. 932 s.v. Astyanax Nr. 18 (Odette Touchefeu); LIMC VII, Zürich und München 1994, S. 519 s. v. Priamos Nr. 124 (Jenifer Neils); Anderson (1997) [Anm. 52], S. 229-31 Abb. 7; Giuliani (2003) [Anm. 52], S. 215-18 Abb. 44. 

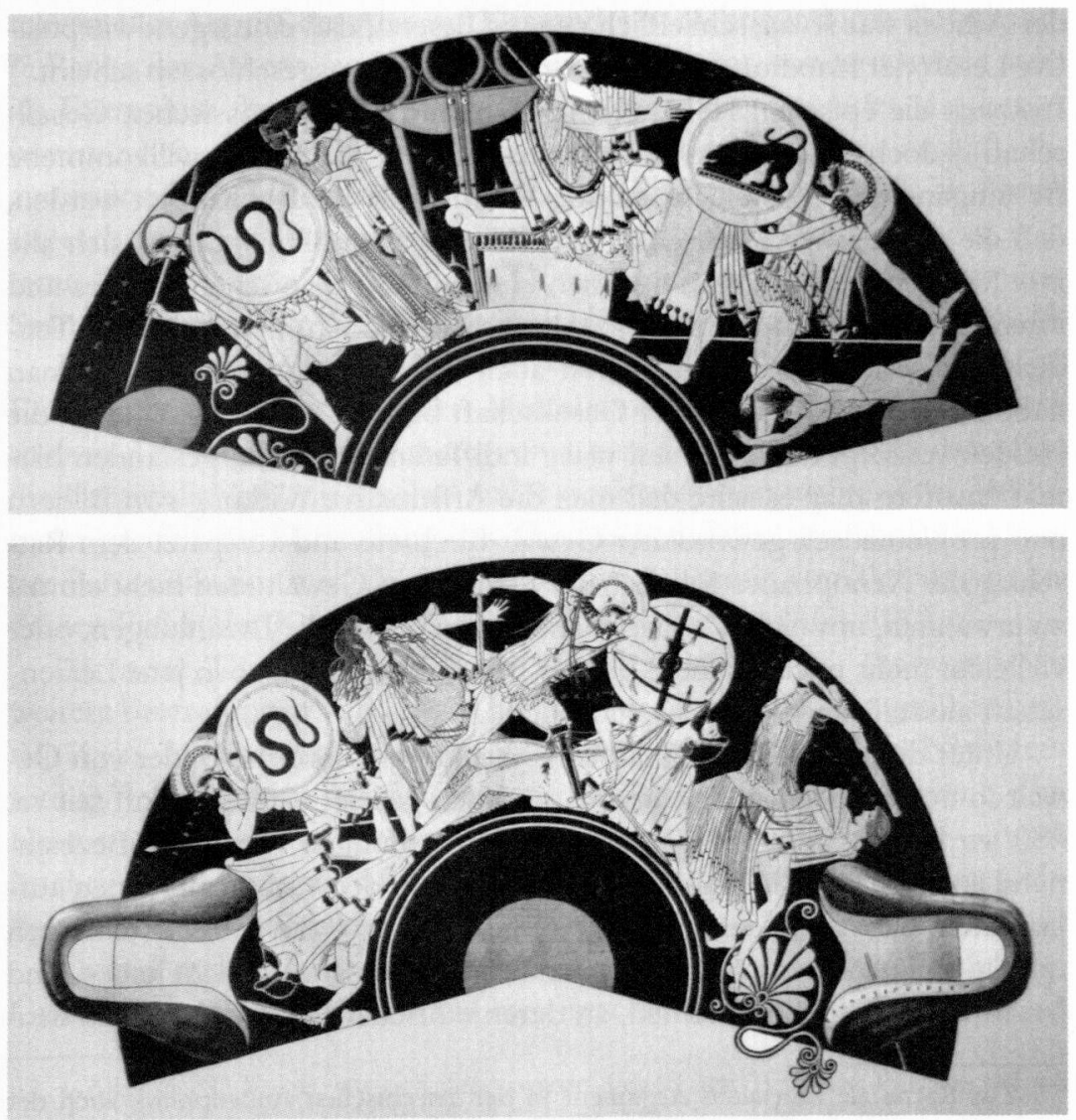

Abb. 9: Schale des Brygos-Malers mit Darstellung der Ilioupersis. Paris, Louvre Inv. G 152 .

schließlich die Vernichtung der Trojaner gezeigt wird - hier sogar ohne die Ajas-Kassandra-Szene - so hängt es allein von der Disposition des Betrachters ab, ob er diesem Geschehen kritisch gegenübertritt.

In der Darstellung selbst ist wenig zu finden, was eine solche Haltung fördern würde, wenn man einmal vom Mord des Priamos am Altar absieht. Doch gehören solche , milderen‘ Sakrilege zu Kriegen dazu, und die völlige Vernichtung des Feindes war als Rachemaßnahme selbst in der späteren attischen Demokratie keineswegs obsolet, wie etwa die Melier schmerzlich erleben mußten. ${ }^{77}$ Diese Erfahrung der affirmativen Wirkung von Bildern mag mit ein Grund dafür gewesen sein, daß die Griechen Bilder für wirkmächtiger hielten als Sprache. ${ }^{78}$ Der Überfall auf Kassandra im Heiligtum 
der Athena war so ausschließlich negativ besetzt, daß eine irgendwie positive Lesart der Handlung des Ajas von vornherein ausgeschlossen scheint. ${ }^{79}$ Insoweit die epischen Helden den Normen der zeitgenössischen Gesellschaft jedoch weitgehend entsprachen, waren die Bilder willkommene Bestätigung des eigenen Lebensstils. Damit soll nicht behauptet werden, $\mathrm{da} ß$ die archaische Gesellschaft ein ethischer Monolith war und sich alle ihre Mitglieder ständig und mit allen Helden des trojanischen Kriegès und ihren Taten identifizierten. Umgekehtt scheint jedoch eben wegen der Tendenz von Bildern zur Affirmation auch die Vorstellung schwierig, man habe dieselben Bilder in einer Gesellschaft benutzt, welche den durch die Helden verkörperten Normen völlig indifferent gegenübergestanden hätte. ${ }^{80}$ Insofern mag es sein, daß man die Affirmative Wirkung von Bildern nun problematisch gewordener Gewalt fürchtete und verspätet dem Ratschlag des Xenophanes folgte, beim Symposion Gewalttaten nicht einmal zu erwähnen, um nicht zu diesen anzuregen. ${ }^{81} \mathrm{Wie}$ die Erzählungen, oder vielleicht mehr noch als diese bergen Bilder die Gefahr, eben jene Leidenschaft auszulösen, welche sie darstellen.

Unter diesem Gesichtspunkt ist aufschlußreich, welche Bilder von Gewalt in der klassischen Zeit überhaupt noch gezeigt werden, denn seit ca. 480 wird keineswegs völlig auf solche Darstellungen verzichtet. Bezeichnend ist vor allem die Wahl der Themen, die in der Vasenmalerei neu aufkommen. Sie vermeiden gerade jene Komplexität, die die Darstellungen um die und nach der Jahrhundertwende so interessant gemacht haben, und beschränken sich auf Themen, an deren ethischer Position kaum zu deu-

77 Zur Rache als zentralem Argument in der griechischen Außenpolitik auch der klassischen Zeit s. Gehrke (1987) [Anm. 12].

78 Herwig Blum: Die antike Mnemotechnik. Spudasmata XV, Hildesheim/New York 1969, bes. S. 164-71; Luca Giuliani: Bilder nach Homer. Vom Nutzen und Nachteil der Lektüre für die Malerei, Freiburg 1998, bes. S. 127-36.

79 Vgl. hier Anm. 82; anders Mangold (2000) [Anm. 2], S. 34-62, bes. S. 61-62, und Moraw (2003) [Anm. 67], S. 339, die vor allem in den späteren Bildern in erster Linie einen männlichen Voyeurismus befriedigt sehen wollen, während der Frevel in den Hintergrund trete.

80 Ich gehe dabei stillschweigend davon aus, daß Bilder niemals, reine Erzählungen ${ }^{6}$ und, reine Unterhaltung ${ }^{c}$ sein können und verweise auf die zahlreichen Bilder, welche nicht eigentlich spannende Schlüsselszenen der Mythen darstellen.

81 Fr. 1 West; vgl. auch Anakr. eleg. 2 West, Stesich. F 210 Page, wo es aber eher darum geht, sich beirn Symposion vergnüglichen Dingen zuzuwenden. In diesem Zusammenhang ist vielleicht nicht unbedeutend, daß sich die Mehrzahl der fraglichen Bilder der atchaischen Zeit auf Symposionsgeschirr befindet und das Symposion offenbar ein bevorzugter Ort hybrider, u. U. sogat zur stasis führender Gewalt war: Fischer (2000) [Anm. 37], bes. S. 93-101. 
teln ist: Der Angriff des Ajas auf Kassandra ist nicht nur durch die bekannte Rache der Athena eindeutig als Frevel bestimmt, sondern wurde auch als Exemplum für frevelhaftes Verhalten schlechthin genutzt, das besser unverzüglich zu bestrafen sei, um Unheil von der Gemeinschaft abzuwenden. ${ }^{82} \mathrm{Daß}$ der Mord an Priamos und Astyanax ebenfalls als Frevel angesehen wurde, da er an einem Altar geschah, ${ }^{83}$ wird zudem in den späteren Darstellungen oft durch die Kombination mit der Ajas-KassandraSzene auf demselben Gefäß oder sogar im selben Bildfeld verdeutlicht. ${ }^{84}$

Eindeutig als Frevel ausgewiesen wird auch die Tat des Tydeus, der im Exzeß den Kopf seines Gegners Melanippos spaltet, um sein Hirn zu verspeisen. Die angewiderte Athena verweigert dem Verwundeten daraufhin die Unsterblichkeit, die sie ihm zuvor noch verleihen wollte. Zwei Vasenmaler greifen zur Darstellung des Themas auf das ungewöhnliche Mittel der Personifikation zurück: Athena geleitet Athanasia von Tydeus weg, zu dessen Füßen der Kopf des Melanippos zu sehen ist (Abb. 10). ${ }^{85}$

Als neue Themen treten im mittleren Drittel des 5 . Jhs, vor allem weitere Bestrafungen von Hybris hinzu, in der Form z.T. von größter Brutalität, in der Sache aber zweifellos akzeptiert, wie etwa die Niobidentötung. ${ }^{86}$ Auch wenn auf den Fragmenten einer Schale des späten 5. Jhs. Kratos und Bia (l) den Frevler Ixion bestrafen, so ist dies im Bild - durch inschriftlichen Verweis auf die gebrochene Gastfreundschaft - als berechtigte Vergeltung geschildert. ${ }^{87}$

Diese Beschränkung auf relativ wenige, ethisch eindeutige Bilder von Gewalttaten steht in deutlichem Gegensatz zur Behandlung des Themas in

82 s. Alk. (fr. 298 Voigt), wo sich der Vorwurf gegen den Tyrannen Pittakos richtet; dazu Wolfgang Rösler: Dichter und Gruppe, München 1980, S. 204-21; vgl, auch Wholfgang Rösler: Der Frevel des Aias in der ,Tlioupersis‘. In: Zeitschrift für Papytologie und Epigraphik 69 (1987) S. 1-8. Der Bezug auf Pittakos ist nicht eindeutig; in jedem Fall ist der Fall Ajas für Alkaios aber ein Paradigma für den Schaden, der einer Gemeinschaft entsteht, wenn sie einen Frevler nicht rechtzeitig bestraft: s. Ferrati (2000) [Anm. 58], S. 129 mit Anm. 42.

83 von den Hoff (2005) [Antn. 5], S. 240-42.

84 Anderson (1997) [Anm. 52], S. 217-29.

85 Shapiro (1993) [Anm. 16], S. 34-36; Borg (2002) [Anm. 16], S. $146-47$.

86 LIMC VI, Zürich und München 1992, S. 916.924 s. v. Niobidai Nr. 4-9 (Wilfred Geominy) mit berechtigtem Einwand gegen Karl Kerényi: Apollon und Niobe, München und Wien 1980, S. 266, der in den Bildern die menschliche Hilflosigkeit angesichts göttlicher Willkür veranschaulicht sah.

87 Erika Simon: Kratos und Bia. In: Würzburger Jahrbücher 1 (1975), S. 177-86; Shapiro (1993) [Anm. 16], S. 166-67; Borg (2002) [Anm. 16], S. 151-53. Bezeichnend ist auch hier der Unterschied zur Prometheustragödie, in dex der Einsatz von Kratos und Bia als ausgesprochen problematisch geschildert wird. 


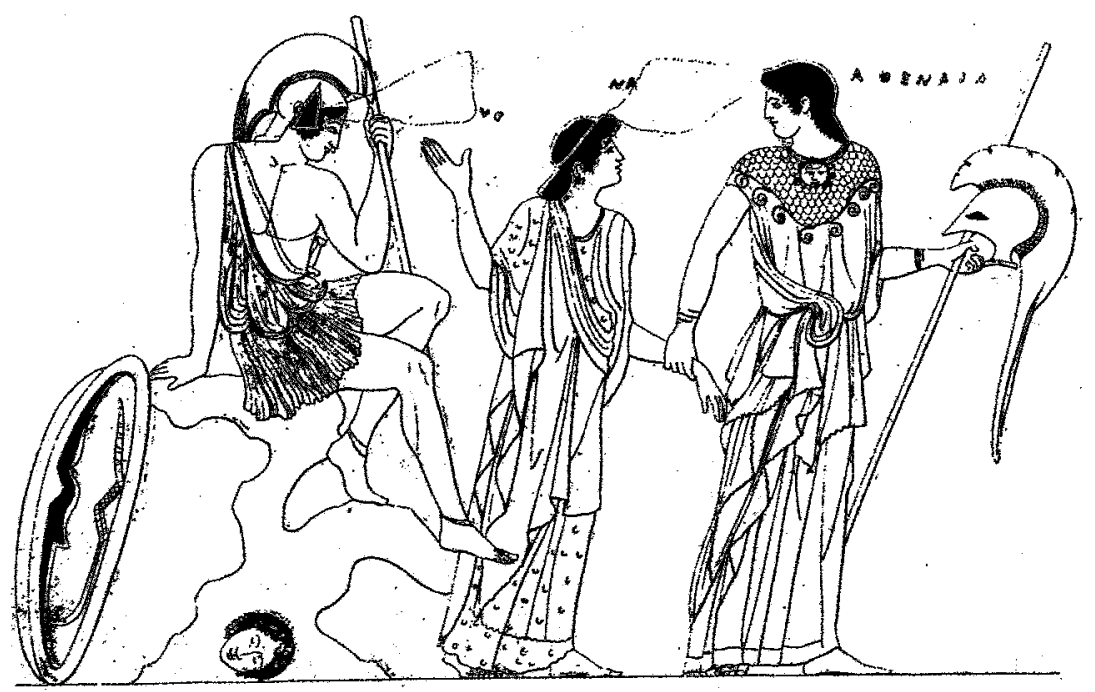

Abb. 10: Glockenkrater des Eupolis-Malers; Athena geleitet Athanasia von Tydeus weg, zu dessen Füßen der Kopf des Melanippos liegt; ehem. Slg. Rosi.

Tragödien derselben Zeit. Dort wird gerade die Ambiguität der Anwendung von Gewalt thematisiert, insbesondere die Tatsache, daß über Akzeptanz oder Ablehnung einer Gewalttat nicht grundsätzlich entschieden werden kann, sondern nur abhängig von der einzelnen Situation und von den Motivationen und Haltungen der Protagonisten. Christopher Gils ,problematischer Heros ${ }^{c}$ entsteht gerade durch das Reflektieren des Heros (und des Zuschauers, Hörers, Lesers) über die praktischen Konsequenzen und die konkrete Umsetzung einer allgemein akzeptierten Norm. ${ }^{88}$ Solche ethischen Reflexionen, oft gar mit offenem Ausgang, sind jedoch mit den Mitteln der klassischen Vasenmalerei kaum darstellbar. Vielleicht hat man auch deshalb im Laufe der Zeit auf die in der Archaik so beliebten Themen verzichtet, ohne diejenigen der Tragödie - um eine möglicherweise naheliegende Option zu nennen - neu aufzugreifen. ${ }^{89}$

88 Christopher Gill: Personality in Greek Epic, Tragedy, and Philosophy. The Self in Dialogue, Oxford 1996, passim.

89 Hier wäre ein ausführlicherer Vergleich zwischen den archaischen und klassischen Themen der Vasenmalerei und denen der Dichtung aufschlußreich. Vorläufig sei nut daran erinnert, daß zwar in fast allen Tragödien Gewalttaten im Zentrum stehen, daß sich diese aber anders als in der archaischen Dichtung in der Regel gegen Blutsverwandte und philoi richtet; bes. Belfiore (2000) [Anm. 8]; auch Seaford (2003) [Anm. 38]. 
Diejenigen Vasenbilder, welche die dem Gewaltdiskurs zugrundeliegenden Besorgnisse vielleicht am besten zum Ausdruck bringen, sind solche der Bestrafung von Dionysos-Frevlern. Sie nehmen unter den Strafaktionen in mehrfacher Hinsicht eine Sonderstellung ein. Zum einen erfolgt die Bestrafung des Frevels hier nicht durch den Geschädigten, die Gottheit, sondern durch Menschen, noch dazu durch nahe Verwandte, die selbst keinen Schaden erlitten haben. Zum zweiten charakterisieren sie die brutale Gewalt zugleich als Auswirkung von Wahnsinn. Wie William Hartis herausgearbeitet hat, besitzen die in der klassischen Literatur üblichen Begriffe für Zorn, orge, cholos und thymos, oft Assoziationen mit Wahnsinn, mania. ${ }^{90}$ Insbesondere in der Tragödie scheint der psychische Zustand, der zu gewalttätigen Reaktionen auf Ehrverletzung und Unrecht führt, in den Blick zu geraten. Er wird nun häufiger mit Begriffen für Wahnsinn oder auch für Krankheit in Verbindung gebracht und damit als vom Willen des Betroffenen weitgehend unabhängig betrachtet. In der Aischyleischen Orestie wird dies durch die Erinnyen zum Ausdruck gebracht, und im Prometheus ist der Zorn als eine Krankheit beschrieben, deren Arzt logoi seien (vv. 375-380). ${ }^{91}$ Spätere Tragödien stellen die Verbindung ebenso her wie gelegentlich Herodot.

Wie sehr Zorn und Gewalt mit Wahnsinn assoziiert werden, können besonders jene Tragödien zeigen, in denen Wahnsinn als Strafe von Göttern, der Hera, der Athena und besonders des Dionysos, ein zentrales Motiv darstellt: Aischylos' Lykurg-Trilogie, die auch den Tod des Orpheus behandelte, seine Dramen Athamas, Pentheus, Xantriai und Bakechen, Sophokles'Ajas, Euripides' Hercules furens und seine Bakchen. Wahnsinn ist dabei gelegentlich die Folge von exzessiver Gewalt, so z.B. bei Orest, meist jedoch ihre Ursache, so in den letztgenannten Beispielen. Indem er als gottgesandt dargestellt wird, ist diese Ursache bezeichnet als etwas außerhalb der Kontrolle des Menschen Stehendes, das darum von um so größerer Gefahr und Zerstörungskraft ist. Insofern scheint der Gewaltdiskurs auch Teil eines Diskurses um Wahnsinn geworden zu sein, der seinerseits aber auch andere Emotionen als wahnsinnigen Zorn, beispielsweise wahnsinnige Iiebesleidenschaft mit umfaßt. So wird man Ruth Padel zustimmen können, wenn sie den Diskurs um maniai als Vorläufer zur dem um die Leidenschaften, die pathe ansieht, deren Gefahrenpotential im 4. Jh. in allen literarischen Genres, besonders

90 Harris (2001) [Anm. 8], S. 50-64; William V. Hartis: The rage of women. In: Braund und Most (2003) [Anm. 25], S. 121-43; s. auch Aristoph., der schon das Waffentragen athenischer Bürger in der Stadt mit Wahnsinn in Verbindung bringt:

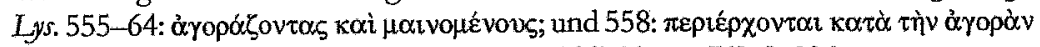

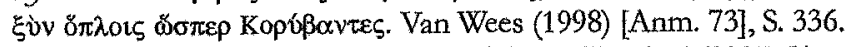

91 Zum Gegensatz zwischen Gewalt und logoi s. Ercolani (2005) [Anm. 8]. 
aber in philosophischen Texten und in Gerichtsreden thematisiert und der sophrosyne gegenübergestellt wird. ${ }^{92}$ Auch im 5 . Jh. wird dem Zorn bereits die sophrosyne als Sinn für das richtige, angemessene Verhalten entgegengesetzt, die einzig die Katastrophen selbstzerstörerischer Leidenschaften verhindern kann. ${ }^{93}$ Insofern besitzen die Bilder von Gewaltexzessen im Zustand des Wahnsinns den Vorzug, eine der Hauptbesorgnisse der klassischen Zeit zu thematisieten und auf den Punkt zu bringen.

Bereits seit Ende des 6. Jhs. erscheint die Zerreißung des Pentheus auf Vasen, die sich auch im späteren 5. Jh. noch mehrfach auf Gefäßen findet. ${ }^{94} \mathrm{Um}$ die Mitte des 5. Jhs. findet sich erstmals der Mord Lykurgs an seiner Familie. ${ }^{95} \mathrm{Im}$ letzten Drittel des 5. Jhs. kommt die Geschichte um die Minyaden neu hinzu, in der die von Dionysos mit mania geschlagene Leukippe ihr eigenes Kind, Hippasos, zerreißt. ${ }^{96}$ Mainaden mit Teilen der von ihnen zerrissenen Tiere finden sich ebenfalls im späteren 5. Jh., in dem

92 Padel (1995) [Anm. 8]; Konstan (2003) [Anm. 39]. Auch Van Wees (1992) [Anm. 12], S. 126, scheint ähnliches zu sehen, wenn er schreibt: „In the epics, anger, not sex, is the dangerous, uncontrollable drive that rules men's lives. Anger, if anything, is what the epics are about." Allerdings müßte man die Bemerkung auf weitere literarische Gattungen bis in die klassische Zeit erweitern. Wenn William Harris meint, die Wurzeln des Interesses an Emotionen und inneren Erfahrungen gingen , mindestens bis in die 450er oder 440er Jahre zurück' und auf Sophokles' Ajas aus den 440er Jahren verweist, so steht dies schon in einem gewissen Gegensatz zu seinen eigenen Ausführungen über die Ilias, die auf erheblich frühere Anfänge der Diskussion verweisen. Zu Atistoteles, der die Gewalttaten der Tragödien ausdrücklich als pathe bezeichnet, s. Belfiore (2000) [Anm. 8], bes. S. 3-6.

93 Dies mag nicht explizit und auch nicht im selben moralphilosophischen Kontext geschehen, doch scheint mir diese Haltung letztlich der Diskussion um den Tyrannenzorn zugrunde zu liegen, der die Tragödien durchzieht, und der Bewertung des Herodot und Thukydides zu entsprechen; dazu Harris (2001) [Anm. 8], S. 157-200; Gabriel Herman: How violent was Athenian society? In: Ritual, Finance, Politics: Athenian Democratic Accounts Presented to David Lewis, hrsgvon Robin Osborne, Oxford 1994, S. 99-117. Sopbrosyne als private Eigenschaft auch in Grabinschriften seit ca. 430: Sally C. Humphreys: Family tombs and tomb cult in ancient Athens. Tradition or traditionalism. In: Journal of Hellenic Studies 100 (1980), S. $114 \mathrm{zu}$ IG i 1058.

94 Dic älteste Darstellung und die erste mit Namensbeischriften findet sich auf einem Psykterfragment des Euphronios Boston, Museum of Fine Arts 10.221a-f. Angelika Schöne: Der Thiasos. Eine ikonogtaphische Untersuchung über das Gefolge des Dionysos in der attischen Vasenmalerei des 6. und 5. Jhs. v. Cht., Göteborg 1987, S. 67-71; LIMC VI, Zürich und München 1994, S. 310-15 s.v. Pentheus Nr. 24. 25. 39-44. 65. 68 (Anneliese Kossatz-Deissmann).

95 LIMC VI, Zürich und München 1994, s.v. Lycourgos I S. 311 Nr. 8; S. 313 N.. 12. 26; S. 317-18 (Alexandre Farnoux). 

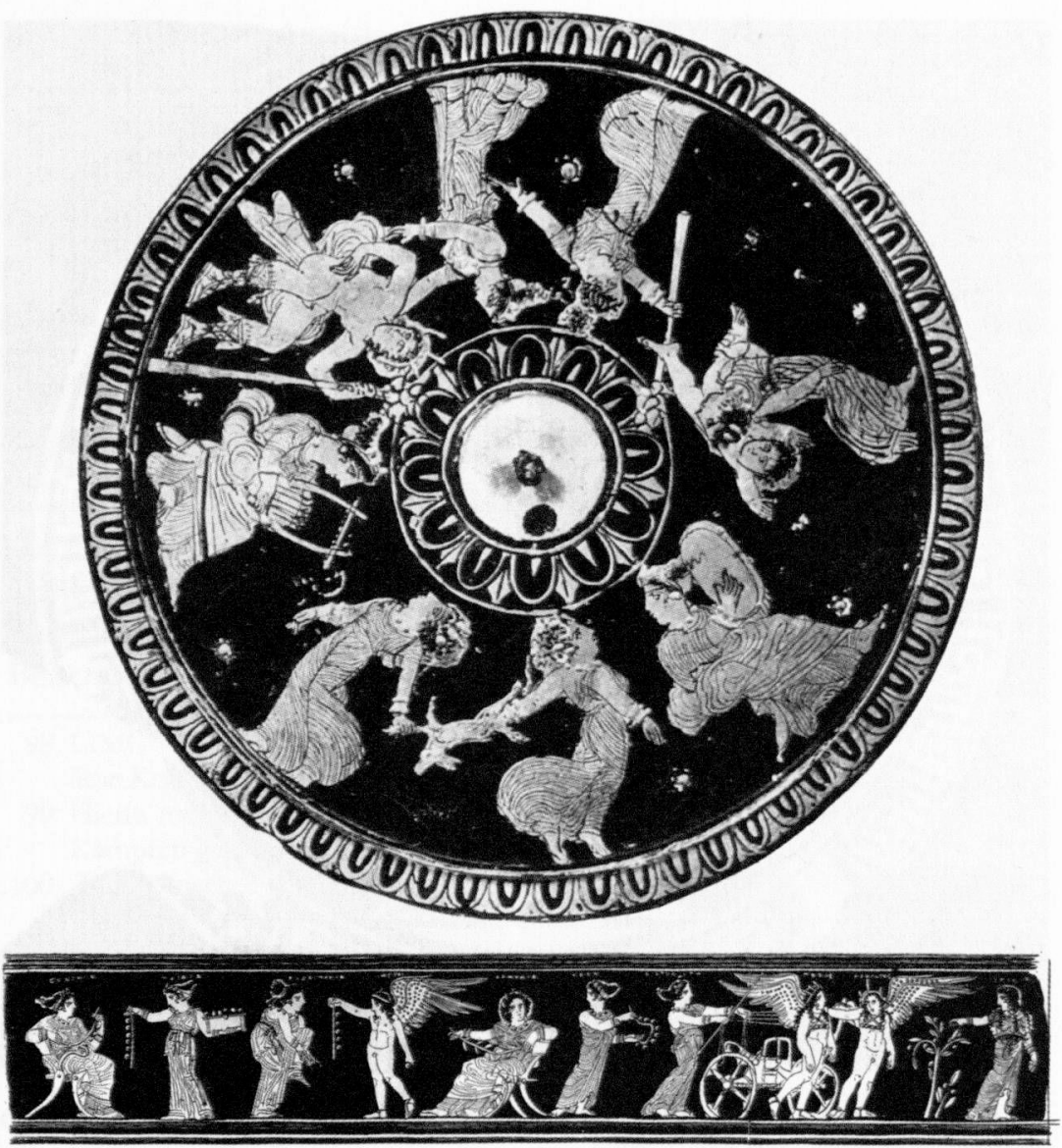

Abb. 11: Pyxix (in der Art) des Meidias-Malers; auf dem Deckel Mainaden, die Tiere und ein Kind zerreißen, auf dem Gefäßkörper eine Allegorie über die Freuden und Grenzen sinnlicher Genüsse. London, British Museum E 775.

die Thiasoi auch allgemein wieder ekstatischer werden. ${ }^{97} \mathrm{Daß}$ es bei den Darstellungen des 5. Jhs. tatsächlich um einen pathe-Diskurs geht, macht vielleicht am besten eine Pyxis in London deutlich (Abb. 11).

96 LIMC VI, Zürich und München 1994, S. 313-14 s. v. Pentheus Nr. 65.68 (Anneliese Kossatz-Deissmann); LIMC VIII, Zürich und Düsseldorf 1997, S. 785 s. v. Mainadai Nr. 33; S. 788 Nr. 72; S. 799 (Ingrid Krauskopf und Erika Simon).

97 LIMC VIII (1997) [Anm. 96], S. 783-92. 798-99 (Ingrid Krauskopf und Erika Simon); Schöne (1987) [Anm. 94], passim; Susanne Moraw: Die Mänade in der attischen Vasenmalerei des 6. und 5. Jahrhunderts v. Chr. Rezeptionsästhetische 


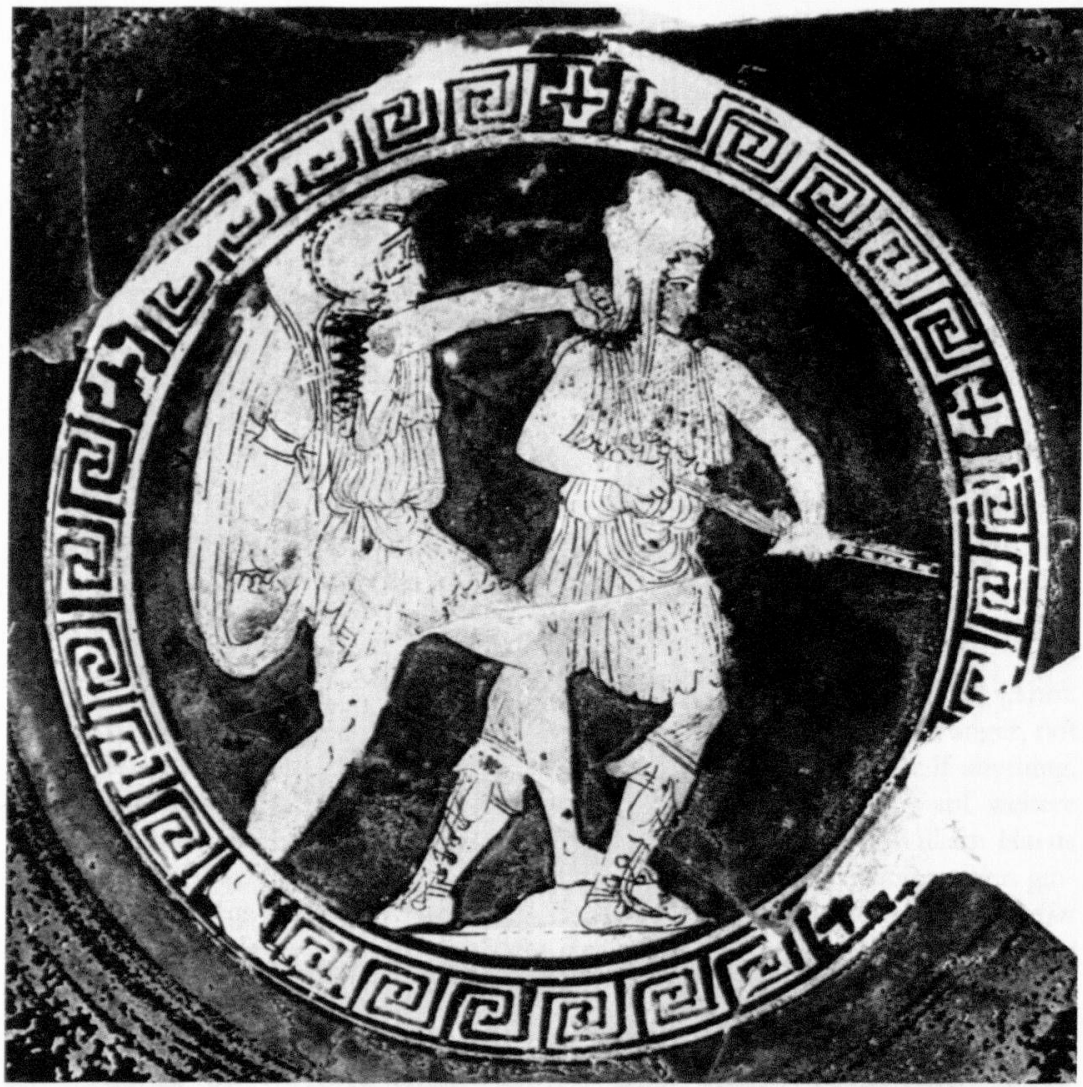

Abb. 12: Schale des Eretria-Malers; Grieche sticht eine Amazone ab. Ferrara, Museo Archeologico Nazionale Inv. T 1039.

Auf ihrem Deckel sind Dionysos und Ariadne (?) dargestellt, sowie zwei Dreiergruppen von Mänaden, deren eine ein Kind über der Schulter schwingt, vermutlich Leukippe mit dem toten Hippasos. Auf dem Körper

Analyse eines antiken Weiblichkeitsentwurfs, Mainz 1998, bes. S. 155-60; 260-1; 263, die die Greueltaten der Mainaden allerdings für ,abstrakte Chiffren für Ekstase“ hält. Da sich die Darstellungen nun auch auf Frauengefäßen finden und sie zudem davon ausgeht, Frauen hätten sich mit den Mänaden identifizieren müssen, fährt sie fort: „Daß diese Chiffren ursprünglich aus einem ganz anderen Kontext - der Visualisierung eines der männlichen Vorstellung entstammenden weiblichen Ungeheuers - stammten u nd dort zudem negativ konnotiert waren, spielte anscheinend keine Rolle.“ (S. 160). 
derselben Pyxis findet sich eine allegorische Darstellung über Freuden und Grenzen erotischer Leidenschaft. ${ }^{98}$

Selbstverständlich dienen diese Darstellungen nicht der ,Erziehung“ des Volkes oder einer politischen Doktrin. Vielmehr scheint es, als spiegele sich in den Vasenbildern nach den Perserkriegen im 5. Jh. die breite Akzeptanz der neuen Polisideologie der klassischen Zeit - oder ist es nur das Bedürfnis nach Affirmation und Selbstvergewisserung? Erst im letzten Jahrhundertdrittel scheinen die Vasenbilder wieder stärker diskursiven Charakter zu erhalten und aktuelle Diskussionsthemen aufzugreifen - wie etwa die um Emotionen und Leidenschaften. ${ }^{99}$ Gewalt scheint dabei durchaus ein wichtiges Element zu sein, jedoch geht es nicht um ein Für oder Wider von Gewalt, sondern - wie im ähnlich gelagerten erotischen Diskurs ${ }^{100}$ um die richtige Anwendung derselben: ${ }^{101}$ im rechten $\mathrm{Maß}$ und ohne übermäßige Leidenschaft - ganz wie der Grieche, der auf den Bildern des Eretriamalers kurz entschlossen und lässig eine Amazone absticht - die Beischrift lautet: kalos (Abb. 12). ${ }^{102}$

98 LIMC VII, Zürich und München 1994, S. 310-15 s. v. Pentheus Nr. 68 (Anneliese Kossatz-Deissmann); zur Deutung Borg (2002) [Anm. 16], S. 190-208.

99 Hierin mag auch ein Grund dafür liegen, daß sich Kentauromachien unter den Kämpfen gegen die Zivilisationsfeinde besonderer Beliebtheit erfreuen.

100 Zum erotischen Diskurs, wie er sich in Vasenbildern spiegelt, s. Borg (2002) [Anm. 16], S. 171-208; mit etwas anderem Ansatz Stähli (1999) [Anm. 74], S. 109-32.

101 Insofern geht die These einer,Auslagerung' von Gewalt in den Mythos (so etwa Günter Fischer und Susanne Moraw: Einleitung. In: Fischer und Moraw 2005 [Anm. 2], S. 12, oder Bentz 2005 [Anm. 3]) am Kern der Sache vorbei: Der Mythos hat hier lediglich den Vorzug, daß der Hintergrund der Ereignisse bzw. die Art der Gegner bekannt sind und somit die ethische Zuordnung des Geschehens vereindeutigt wird.

102 Ferrara T 1039: Adrienne Lezzi-Hafter: Der Eretria-Maler. Werke und Weggefährten, Mainz 1988, S. 314 Kat. 25 Taf. 20. Insofern kann ich die Interpretation von Muth (2005) [Anm. 15], bes. S. 196-97; 199-200, nicht ganz nachvollziehen, die in den Bildern der Zeit nach ca. 470/60, welche meist einen Moment unmittelbat vor dem eigentlichen Schlag darstellen, eine mindestens ebenso große Spannung ausgedrückt sieht wie in denjenigen der Zeit vorher, welche die unmittelbaren Auswirkungen von Gewalt darstellen. Die wohl in einem realen Kampf: tatsächlich höchste Spannung in einem solchen Moment ist in den Bildern in aller Regel nicht dargestellt, weder durch Gesten noch durch Blicke oder Mimik. Vielmehr zeigen sich die Protagonisten in derselben seltsamen Unbeteiligtheit, welche die Bilder der Zeit ab ca. 460 generell kennzeichnet und zu vielerlei Erklärungen Anlaß gegeben hat (s. Kunze 2005 [Anm. 6], S. 45-50 mit weiteren Hinweisen). Diese auffällige Emotionslosigkeit, die im übrigen Muths Analyse einer eher absolut aufgefaßten Uberlegenheit des Siegers entspricht, scheint eher die hier votgetragene These zu bestätigen, daß es bei der Wahl der Ikonographien nicht zuletzt um die Rolle von Pathos und Affekten geht. 